\title{
Nonlinear Stability of Oblate Infinitesimal in Elliptic Restricted Three-Body Problem Influenced by the Oblate and Radiating Primaries
}

\author{
A. Narayan $\left(\mathbb{D},{ }^{1}\right.$ A. Chakraborty $\mathbb{D},{ }^{1}$ and A. Dewangan ${ }^{2}$ \\ ${ }^{1}$ Bhilai Institute of Technology, Durg, C. G. 491001, India \\ ${ }^{2}$ Research Scholar under the Project Approved by CCOST, Endt. no.2260/CCOST/MRP/2015, India \\ Correspondence should be addressed to A. Narayan; ashutoshmaths.narayan@gmail.com
}

Received 27 November 2018; Accepted 4 February 2019; Published 27 February 2019

Academic Editor: Elmetwally Elabbasy

Copyright (C) 2019 A. Narayan et al. This is an open access article distributed under the Creative Commons Attribution License, which permits unrestricted use, distribution, and reproduction in any medium, provided the original work is properly cited.

This work deals with the nonlinear stability of the elliptical restricted three-body problem with oblate and radiating primaries and the oblate infinitesimal. The stability has been analyzed for the resonance cases around $\omega_{1}=2 \omega_{2}$ and $\omega_{1}=3 \omega_{2}$ and also the nonresonance cases. It was observed that the motion of the infinitesimal in this system shows instable behavior when considered in the third order resonance. However, for the fourth order resonance the stability is shown for some mass parameters. The motion in the case of nonresonance was found to be unstable. The problem has been numerically applied to study the movement of the infinitesimal around two binary systems, Luyten-726 and Sirius.

\section{Introduction}

The study of equilibrium points and their stability in restricted three-body problems has attracted the attention of many researchers in the past century, as the stable and unstable resonant motions explain many of the celestial phenomena. The nonlinear stability in circular and elliptical restricted three-body problem was studied in detail by many authors. Markeev [1] employed numerical and analytic methods to study the stability of equilibrium points and periodic motions of nonlinear Hamiltonian systems in cases of resonance. Gyorgyey [2] studied the nonlinear stability of motions around the triangular equilibrium point $L_{5}$. The work was further elaborately studied by various authors ([3-6], et al.) taking into account various other perturbing forces. Ferraz-Mello [7] used the averaging of the elliptic asteroidal problem to study the first order resonance. Henrard \& Caranicolas [8] and Henrard [9] used the perturbation method to study the resonance. Further [10-16] and many others extended the work and explored various aspects of the problem.
In order to investigate the stability of the triangular liberation points the Hamiltonian is simplified by applying Birkhoff's transformation. The normalization method adopted is outlined as follows:

(i) The quadratic form $\mathrm{H}_{2}$ should be reduced so that it corresponds to the normal oscillations modes. This transformation is performed by means of real, linear, and canonical changes of variables.

(ii) After the quadratic part $\mathrm{H}_{2}$ has been reduced to normal form, a nonlinear $2 \pi$ periodic Birkhoff transformation is required to suppress the third-degree term $\mathrm{H}_{3}$.

(iii) The final step is obtaining a Hamiltonian function normalized to fourth order terms obtained by simplifying $\mathrm{H}_{4}$ by means of a canonical Birkhoff transformation.

If $\mathrm{H}_{2}$ is a function of definite sign, then by the virtue of Liapunov's theorem the equilibrium is stable. Otherwise, if $\mathrm{H}_{2}$ is not a function of definite sign, then the stability is 
investigated by means of Arnold's theorem given by the following.

Let the Hamiltonian satisfy the three conditions:

(1) The characteristic equation of the linearized system has pure imaginary roots are $\pm \iota \omega_{1}, \pm \iota \omega_{2}$.

(2) The frequencies $\omega_{1}, \omega_{2}$ satisfy the inequalities $k_{1} \omega_{1}+$ $k_{2} \omega_{2} \neq 0$ for $0<\left|k_{1}\right|+\left|k_{2}\right| \leq 4$, where $k_{1}$ and $k_{2}$ are integers.

(3) The inequality $C_{20} \omega_{1}^{2}+C_{11} \omega_{1} \omega_{2}+C_{02} \omega_{1}^{2} \neq 0$ is fulfilled.

If the above three conditions are satisfied, then the equilibrium points are stable.

The above-mentioned methodology has been used to investigate the nonlinear stability of the elliptic restricted three-body problem with bigger and smaller primaries and infinitesimal as oblate spheroid and also both the primaries as source of radiation. The paper is divided into following sections. Section 1 gives general introduction. The equations of motion are presented in Section 2, and also the triangular equilibrium points are obtained. Existence of resonance in circular case is briefly discussed in Section 3. The normalization of the Hamiltonian is done in Section 4. The second order terms are normalized retaining the third and fourth order terms by using a linear canonical transformation of variables. The stability in third and fourth order resonances is analyzed in Sections 5 and 6, respectively. The stability in nonresonance case is dealt in Section 7 of this paper. The stability of the system has been analyzed using the KAM theorem. The equations used in the intermediate calculation in the sections are given in the Appendix. Numerical applications are presented in Section 8. The discussion and conclusion are drawn in Section 9.

\section{Equation of Motion and Existence of Triangular Points}

The differential equation governing the motion of the oblate infinitesimal mass under the radiation and oblateness of the primaries is represented as follows [17]:

$$
\begin{aligned}
& x^{\prime \prime}-2 y^{\prime}=\phi(\mathrm{e}, \mathrm{f}) \frac{\partial U}{\partial x} \\
& y^{\prime \prime}+2 x^{\prime}=\phi(\mathrm{e}, \mathrm{f}) \frac{\partial U}{\partial y}
\end{aligned}
$$

where

$$
\begin{aligned}
\mathrm{U} & =\frac{x^{2}+y^{2}}{2}+\frac{1}{n^{2}}\left\{q_{1}(1-\mu)\left(\frac{1}{r_{1}}+\frac{A_{1}}{2 r_{1}^{3}}\right)\right. \\
& \left.+q_{2} \mu\left(\frac{1}{r_{2}}+\frac{A_{1}}{2 r_{2}^{3}}\right)+\frac{A_{3}}{2}\left(\frac{1-\mu}{r_{1}^{3}}+\frac{\mu}{r_{2}^{3}}\right)\right\}
\end{aligned}
$$

$$
\begin{aligned}
& \mathrm{r}_{1}^{2}=(x+\mu)^{2}+y^{2} \\
& \mathrm{r}_{2}^{2}=(x+\mu-1)^{2}+y^{2} \\
& \phi(\mathrm{e}, \mathrm{f})=\frac{1}{1+e \cos f} \\
& n^{2}=\frac{1}{a^{3}}\left(1+\frac{3}{2}\left(A_{1}+A_{2}+e^{2}\right)\right)
\end{aligned}
$$

Here prime $\left({ }^{\prime}\right)$ denotes the differentiation with respect to the true anomaly $f . U_{x}$ and $U_{y}$ denote the partial differentiation of $U$ with respect to $x$ and $y$, respectively. $a$ and $e$ represent the semimajor axis and eccentricity of the elliptic path followed by the two primaries. $A_{1}, A_{2}$, and $A_{3}$ are the oblateness parameter of the primaries and infinitesimal, respectively. $q_{1}, q_{2}$ are the radiation factors of the primaries, respectively.

The coordinates of the triangular equilibrium points $(u, v)$ in linear terms of the perturbing forces are given as follows:

$$
\begin{aligned}
u= & \frac{1}{2}-\mu+\left(\frac{1}{2}-2 \delta\right) A_{2}-A_{3}+\left(\frac{-1}{3}+\frac{2 \delta}{3}\right) \beta_{1} \\
& +\left(\frac{1}{3}-\frac{2 \delta}{3}\right) \beta_{2} \\
v= & \frac{\sqrt{3}}{2}\left(1-\frac{5}{3} \delta+\frac{2}{3} e^{2}(-1+2 \delta)-\frac{A_{1}}{3}+\frac{A_{2}}{3}\right. \\
& +\left(-\frac{1}{3}+\frac{10}{9} \delta\right) \beta_{1}+\left(-\frac{2}{9}+\frac{4}{9} \delta\right) \beta_{2}
\end{aligned}
$$

Here $\delta=1-a, \beta_{i}=1-q_{i}, i=1,2$.

The Lagrangian equation of motion of the problem is written as follows:

$$
\begin{aligned}
L= & \frac{1}{2}\left(\dot{x}^{2}+\dot{y}^{2}\right)+\dot{y} x-\dot{x} y+\frac{1}{1+e \cos f}[(1-\mu) \\
& \cdot\left\{\frac{r_{1}^{2}}{2}+\frac{1}{n^{2}}\left(\frac{q_{1}}{r_{1}}+\frac{q_{1} A_{1}+A_{3}}{2 r_{1}^{3}}\right)\right\} \\
& \left.+\mu\left\{\frac{r_{2}^{2}}{2}+\frac{1}{n^{2}}\left(\frac{q_{2}}{r_{2}}+\frac{q_{2} A_{2}+A_{3}}{2 r_{2}^{3}}\right)\right\}\right]
\end{aligned}
$$

Hence, the perturbed Hamiltonian function of the problem is given by

$$
\begin{aligned}
H & =-\frac{p_{x}^{2}+p_{y}^{2}}{2}-p_{y} x+p_{x} y+\frac{x^{2}+y^{2}}{2} \\
& -\frac{1}{1+e \cos f}[(1-\mu)
\end{aligned}
$$




$$
\begin{aligned}
& \cdot\left\{\frac{r_{1}^{2}}{2}+\frac{1}{n^{2}}\left(\frac{q_{1}}{r_{1}}+\frac{q_{1} A_{1}+A_{3}}{2 r_{1}^{3}}\right)\right\} \\
& \left.+\mu\left\{\frac{r_{2}^{2}}{2}+\frac{1}{n^{2}}\left(\frac{q_{2}}{r_{2}}+\frac{q_{2} A_{2}+A_{3}}{2 r_{2}^{3}}\right)\right\}\right]
\end{aligned}
$$

where $p_{x}$ and $p_{y}$ are the generalized components of momentum. The nature of motion near the two equilibrium points will be the same as the two triangular equilibrium solutions are symmetrical to each other. Hence, we consider the motion near the equilibrium point $L_{4}$ for further calculations. To study the stability near this equilibrium point, we shift the origin to $L_{4}$ by the change of variables given by

$$
\begin{aligned}
x & =u+q_{1} ; \\
y & =v+q_{2} ; \\
p_{x} & =p_{u}+p_{1} \\
\text { and } p_{y} & =p_{v}+p_{2}
\end{aligned}
$$

where $(u, v)$ denotes the triangular equilibrium point $L_{4}$ and

$$
\begin{aligned}
& p_{u}=-v, \\
& p_{v}=u
\end{aligned}
$$

\section{Characteristic Roots and Existence of Resonance in Circular Case}

Restricting the Hamiltonian to $\mathrm{H}_{2}$ alone, the characteristic equation is obtained as [18]

$$
\lambda^{4}+\left(4-A^{*}-C^{*}\right) \lambda^{2}+A^{*} C^{*}-B^{* 2}=0
$$

where

$$
\begin{aligned}
A^{*} & =\frac{3}{4}-2\left(\frac{7 e^{2}}{8}(1-2 \mu)+\frac{\beta_{1}}{4}(1-3 \mu)\right. \\
& -\frac{\beta_{2}}{4}(2-3 \mu)-\frac{3 A_{1}}{4}\left(1-\frac{7}{4} \mu\right)+\frac{3 A_{2}}{4}\left(1-\frac{7}{4} \mu\right) \\
& \left.+\frac{\delta}{32}-140+\frac{9 A_{3}}{8}\left(1-\frac{7}{3} \mu\right)\right) \\
B^{*} & =\frac{3 \sqrt{3}}{4}(1-2 \mu)-\sqrt{3}\left[-\frac{11}{4} e^{2}\left(1-\frac{20}{11} \mu\right)\right. \\
& +\frac{\beta_{1}}{6}(1+\mu)-\frac{\beta_{2}}{6}(2-\mu)-\frac{5 \delta}{3}(1-2 \mu) \\
& -\frac{A_{1}}{2}\left(7-\frac{59}{4} \mu\right)-A_{2}\left(2-\frac{29}{8} \mu\right) \\
& \left.-\frac{9 A_{3}}{4}\left(1-\frac{5}{3} \mu\right)-\frac{29 \delta}{16}\left(1-\frac{38}{29} \mu\right)\right] \\
& +\frac{3 A_{2}}{4}\left(3-\frac{11}{4} \mu\right)-\frac{\delta}{32}(95-220 \mu) \\
C^{*} & =\frac{9}{4}+2\left(\frac{e^{2}}{8}(23-22 \mu)+\frac{\beta_{1}}{4}(1-3 \mu)\right. \\
& \\
& \left.=\frac{3 \mu)+\frac{3 A_{1}}{4}\left(3+\frac{11}{4} \mu\right)}{4}(1-\mu)\right) .
\end{aligned}
$$

Assume the frequencies $\omega_{1}$ and $\omega_{2}$, are given by the relation $\omega_{1}^{2}=-\left\{\lambda_{1,2}^{(0)}\right\}^{2}$ and $\omega_{2}^{2}=-\left\{\lambda_{3,4}^{(0)}\right\}^{2}$, where $\lambda_{1,2,3,4}^{(0)}$ are the roots of the characteristic equation (11), when $e=0$. The values are obtained as

$$
\begin{aligned}
& \left(\omega_{1,2}\right)^{2}=\frac{1}{2}\left[1 \pm\left\{1-27 \mu(1-\mu)\left(1+\frac{2}{9} \beta_{1}+\frac{2}{9} \beta_{2}+\frac{94}{9} \delta+\frac{119}{6} A_{1}+\frac{61}{6} A_{2}+17 A_{3}\right)\right\}^{1 / 2}\right. \\
& \left.\quad \times\left(1-\frac{13}{4} \delta-6 A_{1}-3 A_{2}-6 A_{3}\right)\right] .
\end{aligned}
$$

Figures 1-3 show the correlation between $\omega$ and $\mu$ for varying values of the oblateness of the infinitesimal. For the figures, the following values of the perturbing factors are taken: $\beta_{1}=$ $\beta_{2}=0.01, A_{1}=A_{2}=0.0001$, and $\delta=0.001$.

In order to discuss the existence of resonance, firstly we consider the case when $\omega_{1}=\omega_{2}$. Solving (13) for the case, we obtain

$$
\begin{aligned}
1- & 27 \mu(1-\mu)\left(1+\frac{2}{9} \beta_{1}+\frac{2}{9} \beta_{2}+\frac{94}{9} \delta+\frac{119}{6} A_{1}\right. \\
+ & \left.\frac{61}{6} A_{2}+17 A_{3}\right)=0
\end{aligned}
$$

Solving the above equation for value of $\mu<1 / 2$, we get that the region of stability defined by first approximation is

$$
\begin{aligned}
0 & <\mu<\frac{1}{2}-\frac{\sqrt{69}}{18}\left(1+\frac{4}{9} \beta_{1}+\frac{4}{9} \beta_{2}+\frac{188}{9} \delta+\frac{119}{3} A_{1}\right. \\
& \left.+\frac{61}{3} A_{2}+34 A_{3}\right)
\end{aligned}
$$

Thus, the value of $\mu$ admisible for stable equilibrium point for the case $\omega_{1}=\omega_{2}$, when $e=0$, is given as 


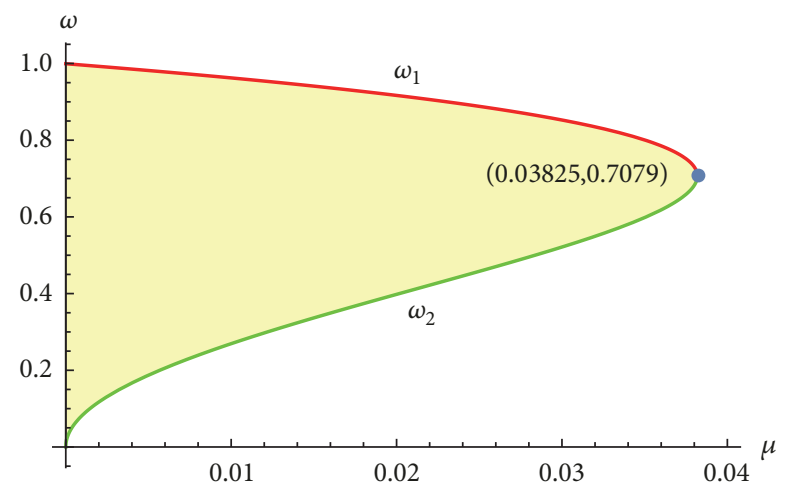

FIgURE 1: Correlation between frequency and mass ratio for $A_{3}=0$.

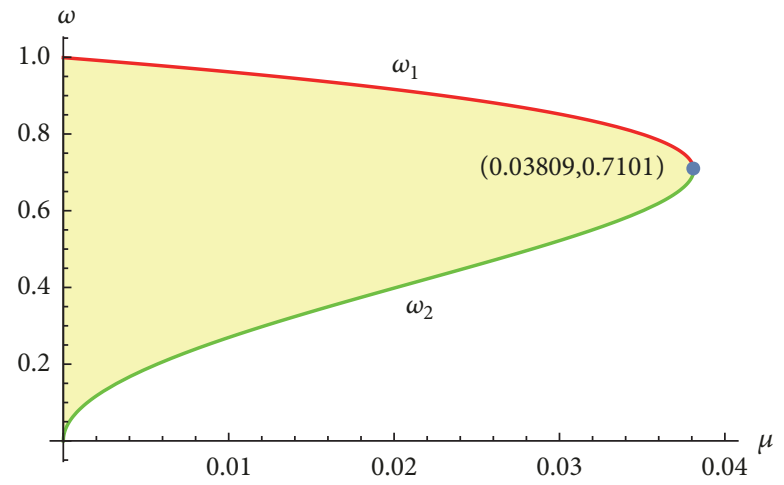

FIgURE 2: Correlation between frequency and mass ratio for $A_{3}=0.0001$.

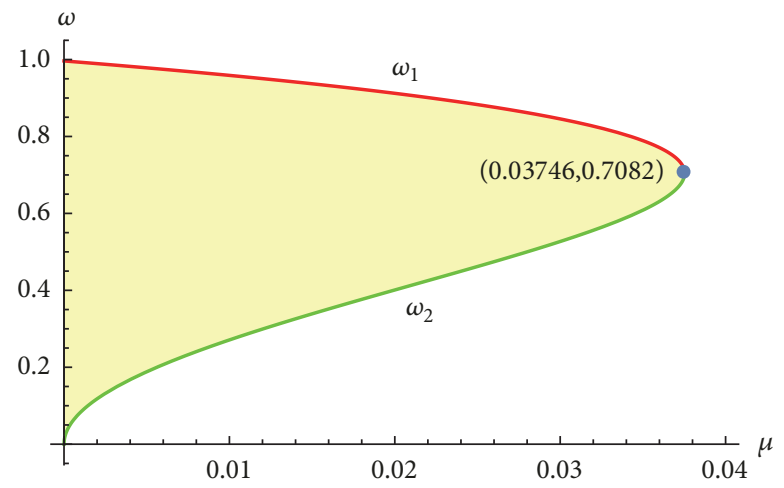

Figure 3: Correlation between frequency and mass ratio for $A_{3}=0.01$.

$$
\begin{aligned}
\mu^{(0)}= & 0.0385209-0.419121 \delta-0.795884 A_{1} \\
& -0.407974 A_{2}-0.682187 A_{3}-0.00891747 \beta_{1} \\
& -0.00891747 \beta_{2}
\end{aligned}
$$

Following the similar procedure, we obtain the critical value of $\mu$ when $\omega_{1}=2 \omega_{2}$ and $\omega_{1}=3 \omega_{2}$ as follows:

$$
\begin{aligned}
\mu^{(02)}= & 0.0242939+0.4941323 \mathrm{~A}_{1}-0.2532947 \mathrm{~A}_{2} \\
& -0.4235419 \mathrm{~A}_{3}-0.2602153 \delta \\
& -0.0055365 \beta_{1}+0.0055365 \beta_{2}
\end{aligned}
$$

$$
\begin{aligned}
\mu^{(03)}= & 0.013516+0.2717915 \mathrm{~A}_{1}-0.1393217 \mathrm{~A}_{2} \\
& -0.23296416 \mathrm{~A}_{3}-0.1431283 \delta \\
& -0.0030453 \beta_{1}+0.0030453 \beta_{2}
\end{aligned}
$$

\section{Normalization of the Hamiltonian}

The Hamiltonian given by (8) is expanded about the Lagrangian point given by (6). Neglecting the terms independent of $p_{i}$ and $q_{i}$, we get the following representation of Hamiltonian: 


$$
\begin{aligned}
H & =\frac{q_{1}^{2}+q_{2}^{2}}{2}-p_{2} q_{1}+p_{1} q_{2}+\frac{p_{1}^{2}+p_{2}^{2}}{2} \\
& -\frac{1}{1+e \cos f}[(1-\mu) \\
& \cdot\left\{\frac{r_{1}^{2}}{2}+\frac{1}{n^{2}}\left(\frac{q_{1}}{r_{1}}+\frac{q_{1} A_{1}+A_{3}}{2 r_{1}^{3}}\right)\right\} \\
& \left.+\mu\left\{\frac{r_{2}^{2}}{2}+\frac{1}{n^{2}}\left(\frac{q_{2}}{r_{2}}+\frac{q_{2} A_{2}+A_{3}}{2 r_{2}^{3}}\right)\right\}\right]
\end{aligned}
$$

Now, expanding the Hamiltonian function given by (8) in the powers of $p_{i}$ and $q_{i} 1 \leq i \leq 2$, we obtain

$$
\begin{aligned}
& H=\sum_{k=0}^{\infty} H_{K} \\
& H=H_{0}+H_{1}+H_{2}+H_{4}+H_{5}+\cdots .
\end{aligned}
$$

Here, $H_{0}=f\left(u, v, p_{u}, p_{v}\right)=$ constant, $H_{1}=0 . H_{2}, H_{3}$, and $H_{4}$ are expression in second, third, and forth order terms of $p_{i}$ and $q_{i}$.

Now, consider the canonical transformation $\left[q_{1}, q_{2}\right.$, $\left.p_{1}, p_{2}\right]$ which transform into $\left[q_{1}^{\prime}, q_{2}^{\prime}, p_{1}^{\prime}, p_{2}^{\prime}\right]$.

That is,

$$
\begin{aligned}
& {\left[q_{1}, q_{2}, p_{1,} p_{2}\right]=\left[q_{1}^{\prime}, q_{2}^{\prime}, p_{1}^{\prime}, p_{2}^{\prime}\right] N} \\
& N=\left|\begin{array}{cccc}
a_{1} & a_{1} c_{1} & -a_{1} c_{1} & a_{1}\left(1-\omega_{1}^{2} b_{1}\right) \\
a_{2} & a_{2} c_{2} & -a_{2} c_{2} & a_{1}\left(1-\omega_{2}^{2} b_{2}\right) \\
0 & a_{1} b_{1} & a_{1}\left(1-b_{1}\right) & a_{1} c_{1} \\
0 & -a_{2} b_{2} & -a_{2}\left(1-b_{2}\right) & -a_{2} c_{2}
\end{array}\right|
\end{aligned}
$$

Using the canonical transformation, the Hamiltonian in the variables will be of the following form:

$$
\begin{aligned}
H= & \frac{1}{2}\left(p_{1}^{\prime 2}+\omega_{1}^{2} q_{1}^{\prime 2}\right)-\frac{1}{2}\left(p_{1}^{\prime 2}+\omega_{2}^{2} q_{2}^{\prime 2}\right) \\
& +\frac{e \cos f}{1+e \cos f}\left[a q_{2}^{\prime 2}+b p_{2}^{\prime 2}+c p_{2}^{\prime} q_{2}^{\prime}+\cdots\right] \\
\text { or } H= & \frac{1}{2}\left(p_{1}^{\prime 2}+\omega_{1}^{2} q_{1}^{\prime 2}\right)-\frac{1}{2}\left(p_{1}^{\prime 2}+\omega_{2}^{2} q_{2}^{\prime 2}\right) \\
& +\sum_{\alpha+\gamma=3}^{\infty} h_{\alpha_{1} \alpha_{2} \gamma_{1} \gamma_{2}} q_{2}^{\prime \alpha_{2}} q_{1}^{\prime \alpha_{1}} p_{2}^{\prime \gamma_{2}} p_{1}^{\prime \gamma_{1}}
\end{aligned}
$$

where $\alpha=\alpha_{1}+\alpha_{2}, \gamma=\gamma_{1}+\gamma_{2}$.
Here $\omega_{1}^{2}=-\left\{\lambda_{1,2}^{(0)}\right\}^{2}$ and $\omega_{2}^{2}=-\left\{\lambda_{3,4}^{(0)}\right\}^{2}$ are the frequencies of the linear system with Hamiltonian $\mathrm{H}_{2}$ and given by the relation:

$$
\begin{aligned}
\lambda^{4} & -\lambda^{2}\left(-1-\frac{\alpha}{4}(13-20 \mu)+6 A_{1}+\frac{3 \mu A_{1}}{2}+3 A_{2}\right. \\
& \left.-\frac{3 \mu A_{2}}{2}+6 A_{3}-3 \mu A_{3}\right)+\frac{27}{4} \mu(1-\mu)\left[1+\frac{2}{9} \beta_{1}\right. \\
& +\frac{2}{9} \beta_{2}+\frac{71}{18} \alpha+\frac{47}{6} A_{1}+\frac{25}{6} A_{2}+\frac{15}{3} A_{3} \\
& \left.-\frac{1}{\mu}\left(\frac{26}{18} \alpha+\frac{8}{6} A_{1}+\frac{8}{6} A_{2}+\frac{4}{3} A_{3}\right)\right]
\end{aligned}
$$

Equating the similar coefficients of $h_{\alpha_{1} \alpha_{2} \gamma_{1} \gamma_{2}}$ and $H_{\alpha_{1} \alpha_{2} \gamma_{1} \gamma_{2}}$ upto the third order terms, the value can be evaluated in terms of $p_{i}{ }^{\prime}, q_{i}{ }^{\prime}$, which are given in the Appendix.

The next transformation is obtained by making the substitution of variables:

$$
\begin{aligned}
& q_{1}^{\prime}=\frac{1}{2} q_{1}^{\prime \prime}+\frac{\iota}{\omega_{1}} p_{1}^{\prime \prime} \\
& p_{1}^{\prime}=\frac{1}{2} \omega_{1} q_{1}^{\prime \prime}+p_{1}^{\prime \prime} \\
& q_{2}^{\prime}=\frac{1}{2} q_{2}^{\prime \prime}+\frac{\iota}{\omega_{2}} p_{2}^{\prime \prime} \\
& p_{2}^{\prime}=\frac{1}{2} \omega_{2} q_{2}^{\prime \prime}+i p_{2}^{\prime \prime}
\end{aligned}
$$

So that the Hamiltonian of (23) got converted to the form:

$$
\begin{aligned}
H= & \iota \omega_{1} q_{1}^{\prime \prime} p_{1}^{\prime \prime}+\iota \omega_{2} q_{2}^{\prime \prime} p_{2}^{\prime \prime} \\
& +\sum_{\alpha+\beta=3}^{\infty} h_{\alpha_{1} \alpha_{2} \gamma_{1} \gamma_{2}}^{\prime} q_{1}^{\prime \alpha_{1}} q_{2}^{\prime \alpha_{2}} p_{1}^{\prime \gamma_{1}} p_{2}^{\prime \gamma_{2}}
\end{aligned}
$$

where the coefficient of third order terms of $h^{\prime}{ }_{\alpha_{1} \alpha_{2} \gamma_{1} \gamma_{2}}$ depends on $\iota \omega_{1},(i=1,2)$ and $h_{\alpha_{1} \alpha_{2} \gamma_{1} \gamma_{2}}$ which are given in the Appendix. Finally, we apply the Birkhoff's transformation of the form $\left(q_{j}^{\prime \prime}, p_{j}{ }^{\prime \prime}\right)$ to $\left(Q_{j}, P_{j}\right)$ and nullify all the third-degree terms except those giving rise to resonance of the form $\omega_{1}=$ $2 \omega_{2}$. For this, take the generating function of the form:

$$
S=q_{1}{ }^{\prime} P_{1}+q_{2} P_{2}+\varepsilon S_{3}+\varepsilon^{2} S_{4}+\cdots
$$

Choose $S_{3}$ in such a way so that

$$
\begin{aligned}
\overline{H_{3}} & =H_{3}+\sum_{j}\left(\frac{\partial S_{3}}{\partial Q_{j}} \frac{\partial H_{2}}{\partial P_{j}}-\frac{\partial S_{3}}{\partial P_{j}} \frac{\partial H_{2}}{\partial Q_{j}}\right)=0 \\
H_{2}\left(Q_{j}, P_{j}\right) & =\iota \omega_{1} Q_{1} P_{1}+\iota \omega_{2} Q_{2} P_{2}
\end{aligned}
$$

Let

$$
S_{3}=\sum_{\alpha+\gamma=3} g_{\alpha_{1} \alpha_{2} \gamma_{1} \gamma_{2}} Q_{1}^{\alpha_{1}} Q_{2}^{\alpha_{2}} P_{1}^{\gamma_{1}} P_{2}^{\gamma_{2}}
$$

where $g_{\alpha_{1} \alpha_{2} \gamma_{1} \gamma_{2}},(\alpha+\gamma=3), \alpha=\alpha_{1}+\alpha_{2}, \gamma=\gamma_{1}+\gamma_{2}$ are to be determined satisfying (28). 
Let

$$
H_{3}=\sum_{\alpha+\gamma=3} h_{\alpha_{1} \alpha_{2} \gamma_{1} \gamma_{2}}^{\prime} q_{1}^{\prime \alpha_{1}} q_{2}^{\prime \prime \alpha_{2}} p_{1}^{\prime \gamma_{1}} p_{2}^{\prime \gamma_{2}}
$$

Now substituting the values of $H_{2}, H_{3}$ in (28) and (29) and equating the coefficients of like powers of the powers of the variable we obtain

$$
g_{\alpha_{1} \alpha_{2} \gamma_{1} \gamma_{2}}=\frac{i h_{\alpha_{1} \alpha_{2} \gamma_{1} \gamma_{2}}^{\prime}}{\left(\alpha_{1}-\gamma_{1}\right) \omega_{1}+\left(\alpha_{2}-\gamma_{2}\right) \omega_{2}}
$$

\section{Stability in Third Order Resonance}

From (32), it can be observed that substituting different values of $\alpha_{i}, \gamma_{i}(i=1,2)$, where $\alpha+\gamma=3$ and $\omega_{1}=2 \omega_{2}$, the denomination of R.H.S. of (32) vanishes giving rise to resonances for two set of values of $\alpha$ and $\gamma$ rendering $S_{3}$ in determinate. Let

$$
D^{r}=\left(\alpha_{1}-\gamma_{1}\right) \omega_{1}+\left(\alpha_{2}-\gamma_{2}\right) \omega_{2} \text { then for } \omega_{1}=2 \omega_{2}
$$

Case 1. When $\alpha_{1}=1, \alpha_{2}=0, \gamma_{1}=0, \gamma_{2}=2$, we have $D^{r}=0$.

Case 2. When $\alpha_{1}=0, \alpha_{2}=2, \gamma_{1}=1, \gamma_{2}=0$, again we have $D^{r}=0$.

Thus, in resonant case $\omega_{1}=2 \omega_{2}$ using Birkhoff's transformation, it is not possible to cancel $\mathrm{H}_{3}$ of the Hamiltonian. In this case $\mathrm{H}_{3}$ retain two resonant terms with coefficients $h^{\prime}{ }_{1002}$ and $h_{0210}^{\prime}$. Thus, Hamiltonian reduces to the following form:

$$
H=\iota \omega_{1} Q_{1} P_{1}+\iota \omega_{2} Q_{2} P_{2}+h^{\prime}{ }_{1002} Q_{1} P_{2}^{2}+h_{0210}^{\prime} P_{1} Q_{2}^{2},
$$

where $h_{1002}^{\prime}=x_{1002}+\iota y_{1002}$ and $h_{0210}^{\prime}=\left(-\omega_{2}^{2} / 2 \omega_{1}\right)\left(y_{1002}+\right.$ $\left(x_{1002}\right)$

Applying canonical change of variables

$$
\begin{gathered}
Q_{1}=\frac{1}{\left(\omega_{1}\right)^{1 / 2}}\left(Q_{1}^{0}-\iota P_{1}^{0}\right) \\
Q_{2}=\frac{1}{\left(\omega_{2}\right)^{1 / 2}}\left(Q_{2}^{0}-\iota P_{2}^{0}\right) \\
P_{1}=\frac{\left(\omega_{1}\right)^{1 / 2}}{2}\left(-\iota Q_{1}^{0}-P_{1}^{0}\right) \\
P_{2}=\frac{\left(\omega_{2}\right)^{1 / 2}}{2}\left(Q_{2}^{0}-\iota P_{2}^{0}\right)
\end{gathered}
$$

the Hamiltonian equation (34) becomes

$$
\begin{aligned}
H^{0} & =\frac{\omega_{1}}{2}\left(Q_{1}^{02}+P_{1}^{02}\right)-\frac{\omega_{2}}{2}\left(Q_{2}^{02}+P_{2}^{02}\right) \\
& +\left[\frac { \omega _ { 2 } } { 2 ( \omega _ { 2 } ) ^ { 1 / 2 } } \left\{x_{1002}\left(Q_{1}^{0} Q_{2}^{02}-Q_{1}^{0} P_{2}^{02}-2 P_{2}^{0} Q_{2}^{0}\right)\right.\right. \\
& \left.\left.+y_{1002}\left(2 P_{2}^{0} Q_{2}^{0} Q_{1}^{0}+P_{1}^{0} Q_{2}^{02}-P_{1}^{0} P_{2}^{02}\right)\right\}\right]
\end{aligned}
$$

If $x_{1002}^{2}+y_{1002}^{2} \neq 0$, then the canonical transformation in polar coordinates is given by

$$
\begin{aligned}
& Q_{1}^{0}=\left(2 r_{1}\right)^{1 / 2} \sin \left(\phi_{1}-\theta_{1}\right) ; \\
& P_{1}^{0}=\left(2 r_{1}\right)^{1 / 2} \cos \left(\phi_{1}-\theta_{1}\right) ; \\
& Q_{2}^{0}=\left(2 r_{1}\right)^{1 / 2} \sin \left(\phi_{2}\right) ; \\
& P_{2}^{0}=\left(2 r_{1}\right)^{1 / 2} \cos \left(\phi_{2}\right),
\end{aligned}
$$

where $\theta_{1}$ is given as

$$
\begin{aligned}
& \sin \theta_{1}=\frac{y_{1002}}{\left(x_{1002}^{2}+y_{1002}^{2}\right)^{1 / 2}} \\
& \sin \theta_{1}=\frac{x_{1002}}{\left(x_{1002}^{2}+y_{1002}^{2}\right)^{1 / 2}} .
\end{aligned}
$$

Thus, the Hamiltonian will of the form

$$
\begin{aligned}
& \text { H } \\
& =2 \omega_{2} r_{1}-\omega_{2} r_{2} \\
& -\left[\omega_{2}\left(x_{1002}^{2}+y_{1002}^{2}\right)^{1 / 2} r_{2}\left(r_{1}\right)^{1 / 2} \sin \left(\phi_{1}+2 \phi_{2}\right)\right] \\
& +H_{4}^{0}\left(r_{j}, \phi_{j}\right)+\cdots
\end{aligned}
$$

Now, to find the value of $H_{4}$, let us assume $x_{1002}^{2}+y_{1002}^{2}=0$. Then the normalized form of $H_{4}$ is given as

$$
\begin{aligned}
H_{4}= & C_{20}\left(Q_{1}, P_{1}\right)^{2}+C_{11}\left(Q_{1}, P_{1}\right)\left(Q_{2}, P_{2}\right) \\
& -C_{02}\left(Q_{2}, P_{2}\right)^{2}
\end{aligned}
$$

with the help of generating function $S_{4}$ chosen, so that it satisfies

$$
\sum_{j}\left(\frac{\partial S_{4}}{\partial Q_{j}} \frac{\partial H_{2}}{\partial P_{j}}-\frac{\partial S_{4}}{\partial P_{j}} \frac{\partial H_{2}}{\partial Q_{j}}\right)+K_{4}=0
$$

where $K_{4}$ is the nonhomogenous part of (40):

$$
\begin{aligned}
& H_{4}\left(Q_{i}, P_{i}\right)+\sum_{i, j} \frac{\partial S_{3}}{\partial P_{i}} \frac{\partial S_{3}}{\partial Q_{j}} \frac{\partial^{2} H_{2}}{\partial P_{i} \partial Q_{j}} \\
& \quad+\sum_{i, j} \frac{\partial S_{3}}{\partial Q_{j}} \frac{\partial^{2} S_{3}}{\partial P_{i} \partial P_{j}} \frac{\partial H_{2}}{\partial Q_{i}}-\sum_{i, j} \frac{\partial S_{3}}{\partial Q_{j}} \frac{\partial^{2} S_{3}}{\partial Q_{i} \partial P_{j}} \frac{\partial H_{2}}{\partial P_{i}}
\end{aligned}
$$

The coefficients $C_{20}, C_{11}$, and $C_{02}$ are given as 


$$
\begin{aligned}
& C_{20}=\frac{3}{2} \omega_{1}^{2} a_{1}^{4} b_{1}^{4} H_{0400}+\frac{3 a_{1}^{4}}{2 \omega_{1}^{2}}\left(H_{4000}+c_{1} H_{3100}\right. \\
& \left.+c_{1}^{2} H_{2200}+c_{1}^{3} H_{1300}+6 c_{1}^{4} H_{0400}\right)+\frac{a_{1}^{4} b_{1}^{2}}{2}\left(H_{2200}\right. \\
& \left.+3 c_{1} H_{1300}+6 c_{1}^{2} H_{0400}\right)-\frac{3}{8} \omega_{1}^{2}\left(y_{0030}^{2}+x_{0030}^{2}\right) \\
& -\frac{3}{2}\left(y_{1020}^{2}+x_{1020}^{2}\right)-\frac{\omega_{1}^{2}}{8\left(2 \omega_{1}-\omega_{2}\right)}\left(y_{0120}^{2}+x_{0120}^{2}\right) \\
& +\frac{1}{2}\left(y_{1011}^{2}+x_{1011}^{2}\right)+\frac{\omega_{2} \omega_{1}^{2}}{8\left(2 \omega_{1}+\omega_{2}\right)}\left(y_{0021}^{2}+x_{0021}^{2}\right) \\
& C_{11}=6 \omega_{1} \omega_{2} a_{1}^{2} a_{2}^{2} b_{1}^{2} b_{2}^{2} H_{0400}+\frac{a_{1}^{2} a_{2}^{2}}{\omega_{1} \omega_{2}}\left\{6 H_{4000}+3\left(c_{1}\right.\right. \\
& \left.+c_{2}\right) H_{3100}+\left(c_{1}^{2}+4 c_{1} c_{2}+c_{2}^{2}\right) H_{2200}+3 c_{1} c_{2}\left(c_{1}+c_{2}\right) \\
& \left.\cdot H_{1300}+6 c_{1}^{2} c_{2}^{2} H_{0400}\right)+\frac{\omega_{1} a_{1}^{2} a_{2}^{2} b_{1}^{2}}{\omega_{2}}\left(H_{2200}\right. \\
& \left.+3 c_{2} H_{1300}+6 c_{2}^{2} H_{0400}\right)+\frac{\omega_{2} a_{1}^{2} a_{2}^{2} b_{2}^{2}}{\omega_{1}}\left(H_{2200}\right. \\
& \left.+3 c_{1} H_{1300}+6 c_{1}^{2} H_{0400}\right)-\frac{2 \omega_{2}^{2}}{\left(\omega_{1}-2 \omega_{2}\right)}\left(x_{1002}^{2}\right. \\
& \left.+y_{1002}^{2}\right)+\frac{\omega_{1} \omega_{2}^{2}}{2\left(\omega_{1}+2 \omega_{2}\right)}\left(x_{0012}^{2}+y_{0012}^{2}\right) \\
& -\frac{\omega_{2} \omega_{1}^{2}}{2\left(2 \omega_{1}+\omega_{2}\right)}\left(x_{0021}^{2}+y_{0021}^{2}\right) \\
& -\frac{2 \omega_{1}^{2}}{\left(2 \omega_{1}-\omega_{2}\right)}\left(x_{0120}^{2}+y_{0120}^{2}\right)+2\left(x_{0111} x_{1020}\right. \\
& \left.+y_{0111} y_{1020}\right)-\frac{4}{\omega_{2}}\left(x_{0201} y_{1011}+x_{1011} y_{0201}\right) \\
& C_{02}=\frac{3}{2} \omega_{2}^{2} a_{2}^{4} b_{2}^{4} H_{0400}+\frac{3 a_{2}^{4}}{2 \omega_{2}^{2}}\left(H_{4000}+c_{2} H_{3100}\right. \\
& \left.+c_{2}^{2} H_{2200}+c_{2}^{3} H_{1300}+c_{2}^{4} H_{0400}\right)+\frac{a_{2}^{4} b_{2}^{2}}{2}\left(H_{2200}\right. \\
& \left.+3 c_{2} H_{1300}+6 c_{2}^{2} H_{0400}\right)+\frac{3}{8} \omega_{2}^{2}\left(y_{0003}^{2}+x_{0003}^{2}\right) \\
& +\frac{6}{\omega_{2}^{2}}\left(x_{0201}^{2}+y_{0201}^{2}\right)-\frac{\omega_{2}^{2}}{2 \omega_{1}\left(\omega_{1}-2 \omega_{2}\right)}\left(y_{1002}^{2}\right. \\
& \left.+x_{1002}^{2}\right)-\frac{1}{2}\left(x_{0111}^{2}+y_{0111}^{2}\right) \\
& -\frac{\omega_{1} \omega_{2}^{2}}{8\left(\omega_{1}+2 \omega_{2}\right)}\left(x_{0012}^{2}+y_{0012}^{2}\right)
\end{aligned}
$$

Consequently, the Hamiltonian of the dynamical system reduces to the form as

$$
\begin{aligned}
H= & \iota \omega_{1} Q_{1} P_{1}+\iota \omega_{2} Q_{2} P_{2}-C_{20}\left(Q_{1} P_{1}\right)^{2} \\
& +C_{11}\left(Q_{1} P_{1}\right)\left(Q_{2} P_{2}\right)-C_{02}\left(Q_{2} P_{2}\right)^{2}+O|Q|^{5}
\end{aligned}
$$

If $x_{1002}^{2}+y_{1002}^{2}=0$ and $C_{20}+2 C_{11}+4 C_{02} \neq 0$ then by virtue of Markeev's theorem (Markeev 1967) the equilibrium is stable.

\section{Stability in Fourth Order Resonance}

The Hamiltonian $\mathrm{H}$ in this case will be written as

$$
\begin{aligned}
H= & \iota \omega_{1} q_{1}^{\prime \prime} p_{1}^{\prime \prime}+\iota \omega_{2} q_{2}^{\prime \prime} p_{2}^{\prime \prime} \\
& +\sum_{\alpha+\gamma=4} h_{\alpha_{1} \alpha_{2} \gamma_{1} \gamma_{2}}^{\prime} q_{1}^{\prime \alpha_{1}} q_{2}^{\prime \alpha_{2}} p_{1}^{\prime \prime} p_{2}^{\prime \prime \gamma_{2}}+O\left|q^{\prime \prime}\right|^{5}
\end{aligned}
$$

where $\left|q^{\prime \prime}\right|=\left(q_{1}^{\prime \prime}+q_{2}^{\prime \prime}+p_{1}^{\prime \prime}+p_{2}^{\prime \prime}\right)^{1 / 2}$ and $h_{\alpha_{1} \alpha_{2} \gamma_{1} \gamma_{2}}^{\prime}$ depend on $\omega_{i}$ and $h_{\alpha_{1} \alpha_{2} \gamma_{1} \gamma_{2}}$. Now, using Birkhoff's transformation by means of generating function $S$ where $S=S_{2}+S_{3}+S_{4} \ldots$, choose $S_{4}$ such that $\overline{H_{4}}$ takes the normalized form which is given as follows:

$$
\begin{aligned}
\overline{H_{4}}= & -C_{20}\left(Q_{1}, P_{1}\right)^{2}+C_{11}\left(Q_{1}, P_{1}\right)\left(Q_{2}, P_{2}\right) \\
& -C_{02}\left(Q_{2}, P_{2}\right)^{2}
\end{aligned}
$$

where

$$
\begin{aligned}
\overline{H_{4}}= & H_{4}+\sum_{j}\left(\frac{\partial S_{4}}{\partial Q_{j}} \frac{\partial H_{2}}{\partial P_{j}}-\frac{\partial S_{4}}{\partial P_{j}} \frac{\partial H_{2}}{\partial Q_{j}}\right) \\
& +\sum_{i, j} \frac{\partial S_{3}}{\partial P_{i}} \frac{\partial^{2} H_{2}}{\partial P_{i} \partial Q_{j}} \frac{\partial S_{3}}{\partial Q_{j}}+\sum_{i, j} \frac{\partial S_{3}}{\partial Q_{j}} \frac{\partial^{2} S_{3}}{\partial P_{i} \partial P_{j}} \frac{\partial H_{2}}{\partial Q_{i}} \\
& -\sum_{i, j} \frac{\partial S_{3}}{\partial Q_{j}} \frac{\partial^{2} S_{3}}{\partial Q_{i} \partial P_{j}} \frac{\partial H_{2}}{\partial P_{i}}
\end{aligned}
$$

That is,

$$
\sum_{j}\left(\frac{\partial S_{4}}{\partial Q_{j}} \frac{\partial H_{2}}{\partial P_{j}}-\frac{\partial S_{4}}{\partial P_{j}} \frac{\partial H_{2}}{\partial Q_{j}}\right)+K_{4}=0
$$

where $K_{4}$ is the nonhomogeneous part of (45), where homogeneity is considered in terms of product $Q_{i} P_{i}$.

Here,

$$
\begin{aligned}
& S_{3}=\sum_{\alpha+\gamma=3} g_{\alpha_{1} \alpha_{2} \gamma_{1} \gamma_{2}} Q_{1}^{\alpha_{1}} Q_{2}^{\alpha_{2}} P_{1}^{\gamma_{1}} P_{2}^{\gamma_{2}} \\
& H_{2}=\overline{H_{2}}=\iota \omega_{1} Q_{1} P_{1}+\iota \omega_{2} Q_{2} P_{2} \\
& \overline{H_{3}}=0
\end{aligned}
$$

Let $H_{4}=\sum_{\alpha+\gamma=4} h_{\alpha_{1} \alpha_{2} \gamma_{1} \gamma_{2}} Q_{1}^{\alpha_{1}} Q_{2}^{\alpha_{2}} P_{1}^{\gamma_{1}} P_{2}^{\gamma_{2}}$ and $S_{4}=$ $\sum_{\alpha+\gamma=4} g_{\alpha_{1} \alpha_{2} \gamma_{1} \gamma_{2}} Q_{1}^{\alpha_{1}} Q_{2}^{\alpha_{2}} P_{1}^{\gamma_{1}} P_{2}^{\gamma_{2}}$ where $g_{\alpha_{1} \alpha_{2} \gamma_{1} \gamma_{2}}$ are to be determined satisfying (48). Substituting the values in (48) and 
equating the coefficient of similar powers and different nonhomogeneous terms to zero, we have

$$
g_{\alpha_{1} \alpha_{2} \gamma_{1} \gamma_{2}}=\frac{\left.\iota \text { cofficient of } K_{4}\right)}{\left(\alpha_{1}-\gamma_{1}\right) \omega_{1}+\left(\alpha_{2}-\gamma_{2}\right) \omega_{2}}
$$

In the above equation, when substituting different values $\left(\alpha_{i}, \gamma_{i}\right), i=1,2$ and $\omega_{1}=3 \omega_{2}$ the denominator of (50) vanishes for two sets of values of $\alpha$ and $\gamma$ giving rise to resonant terms $l_{1003} Q_{1} P_{2}^{3}$ and $l_{0310} P_{1} Q_{2}^{3}$. Thus, the new Hamiltonian is obtained as

$$
\begin{aligned}
\bar{H} & =\iota \omega_{1} Q_{1} P_{1}+\iota \omega_{2} Q_{2} P_{2}+\left\{l_{1003} Q_{1} P_{2}^{3}+l_{0310} P_{1} Q_{2}^{2}\right. \\
& -C_{20}\left(Q_{1} P_{1}\right)^{2}+C_{11}\left(Q_{1} P_{1}\right)\left(Q_{2} P_{2}\right) \\
& \left.-C_{02}\left(Q_{2} P_{2}\right)^{2}\right\}
\end{aligned}
$$

where $l_{1003}$ and $l_{0310}$ are given by

$$
\begin{aligned}
l_{1003}= & \left\{\frac{\omega_{1}}{2} h_{0013}+\frac{h_{1300}}{2 \omega_{2}^{3}}-\frac{h_{1102}}{2 \omega_{2}}-\frac{\omega_{1} h_{0211}}{2 \omega_{2}^{2}}\right\} \\
& -\frac{2 h_{2001}^{\prime} h_{0012}^{\prime}}{\iota\left(2 \omega_{1}-\omega_{2}\right)}-\frac{3 h^{\prime}{ }_{0003} h_{1101}^{\prime}}{\iota \omega_{1}} \\
& +\frac{2 h_{1002}^{\prime} h_{0102}^{\prime}}{\iota \omega_{2}}-\frac{h_{1011}^{\prime} h_{1002}^{\prime}}{\iota\left(\omega_{1}-2 \omega_{2}\right)} \\
l_{0310}= & \left\{-\frac{\left.\omega_{1 h_{0112}}-\frac{h_{1003}}{2 \omega_{2}}+\frac{h_{1201}}{2 \omega_{2}^{2}}-\frac{\omega_{1} h_{0310}}{2 \omega_{2}^{3}}\right\}}{}\right\} \\
& -\frac{2 h_{0120}^{\prime} h_{1200}^{\prime}}{\iota\left(\omega_{1}+2 \omega_{2}\right)}-\frac{h_{1110}^{\prime} h_{0210}^{\prime}}{\iota \omega_{2}} \\
& +\frac{2 h_{0210}^{\prime} h_{0201}^{\prime}}{\iota\left(\omega_{1}-2 \omega_{2}\right)}-\frac{h_{0300}^{\prime} h_{0111}^{\prime}}{\iota \omega_{2}}
\end{aligned}
$$

where the values $h_{\alpha_{1} \alpha_{2} \gamma_{1} \gamma_{2}}$ are given in the Appendix. Let $l_{1003}=x_{1003}+\iota y_{1003}$, and

$$
l_{1003}=-\frac{\omega_{1}^{2}}{12}\left(x_{1003}-\iota y_{1003}\right)
$$

in which

$$
\begin{aligned}
& x_{1003}=-6 \omega_{2} a_{1} a_{2}^{3} a_{1} a_{2}^{3} H_{0400}+\frac{a_{1} a_{2}^{3}}{2 \omega_{2}^{3}}\left\{4 H_{4000}\right. \\
& +\left(c_{1}+3 c_{2}\right) H_{0400}+2 c_{2}\left(c_{1}+c_{2}\right) H_{2200} \\
& \left.+c_{2}^{2}\left(3 c_{1}+c_{2}\right) H_{1300}\right\}-\frac{a_{1} b_{2}^{2} a_{2}^{3}}{2 \omega_{2}}\left\{2 H_{2200}\right. \\
& \left.+\left(c_{1}+c_{2}\right) H_{1300}+12 c_{1} c_{2} H_{0400}\right\}
\end{aligned}
$$

$$
\begin{aligned}
& +\frac{3 a_{1} b_{1}^{2} b_{2}^{2} a_{2}^{3}}{2 \omega_{2}}\left\{H_{2200}+3 c_{2} H_{1300}+6 c_{2}^{2} H_{0400}\right\} \\
& -\frac{9}{5}\left(x_{0120} x_{0012}+y_{0120} y_{0012}\right)-\frac{1}{\omega_{2}}\left(x_{1002} y_{1011}\right. \\
& \left.+x_{1011} y_{1002}\right)+\frac{4}{\omega_{2}^{2}}\left(x_{1002} x_{0201}+y_{1002} y_{0201}\right) \\
& +\frac{3}{2}\left(x_{0003} x_{0111}+y_{0003} y_{0111}\right) \\
& y_{1003}=-\frac{9}{2} a_{1} a_{2}^{3} b_{1} b_{2}^{2}\left(H_{1300}+4 c_{2} H_{0400}\right) \\
& +\frac{b_{2}^{3} a_{1} a_{2}^{3}}{2}\left(H_{1300}+4 c_{1} H_{0400}\right)-\frac{a_{1} b_{2} a_{2}^{3}}{2 \omega_{2}}\left\{3 H_{3100}\right. \\
& +2\left(c_{1}+2 c_{2}\right) H_{2200}+3 c_{2}\left(2 c_{1}+c_{2}\right) H_{1300} \\
& \left.+12 c_{1} c_{2}^{2} H_{0400}\right\}+\frac{3 a_{1} b_{1} a_{2}^{3}}{2 \omega_{2}^{2}}\left\{H_{3100}+2 c_{2} H_{2200}\right. \\
& \left.+3 c_{2}^{2} H_{1300}+4 c_{2}^{3} H_{0400}\right\}-\frac{9}{5}\left(x_{0120} y_{0012}\right. \\
& \left.+x_{0012} y_{0120}\right)-\frac{1}{\omega_{2}}\left(y_{1011} y_{1002}-x_{1011} y_{1002}\right) \\
& +\frac{4}{\omega_{2}^{2}}\left(x_{0201} y_{1002}-x_{1002} y_{0201}\right)+\frac{3}{2}\left(x_{0111} y_{0003}\right. \\
& \left.-x_{0003} y_{0111}\right)
\end{aligned}
$$

Now, using the transformation (35) and assuming that $x_{1003}^{2}+y_{1003}^{2} \neq 0$, the Hamiltonian reduces to the form

$$
\begin{aligned}
H & =\frac{3}{2} \omega_{2}\left(Q_{1}^{02}+P_{1}^{02}\right)-\frac{\omega_{2}}{2}\left(Q_{2}^{02}+P_{2}^{02}\right)\left\{\frac { 1 } { 4 } C _ { 0 2 } \left(Q_{1}^{02}\right.\right. \\
& \left.+P_{1}^{02}\right)+\frac{c_{11}}{4}\left(Q_{1}^{02}+P_{1}^{02}\right)\left(Q_{2}^{02}+P_{2}^{02}\right) \\
& +\frac{\omega_{2} \sqrt{3}}{12}\left\{P_{2}^{0}\left(P_{2}^{02}-3 Q_{2}^{02}\right)\left(x_{1003} P_{1}^{0}-y_{1003} Q_{1}^{0}\right)\right. \\
& +Q_{2}^{0}\left(Q_{2}^{02}-3 P_{2}^{02}\right)\left(y_{1003} P_{1}^{0}+x_{0003} Q_{1}^{0}\right) \\
& \left.\left.+O|Q|^{5}\right)\right\}
\end{aligned}
$$

Applying transformation in polar coordinates given by (39) where $\theta_{2}=0$ and $\theta_{1}$ is given by the relations:

$$
\begin{aligned}
& \sin \theta_{1}=\frac{x_{1003}}{\left(x_{1003}^{2}+y_{1003}^{2}\right)^{1 / 2}}, \\
& \cos \theta_{1}=\frac{-y_{1003}}{\left(x_{1003}^{2}+y_{1003}^{2}\right)^{1 / 2}} .
\end{aligned}
$$


Hence, the normalized Hamiltonian in the polar form is given by

$$
\begin{aligned}
H= & 3 \omega_{2} r_{1}-\omega_{2} r_{2}+\left[C_{20} r_{1}^{2}+C_{11} r_{1} r_{2} C_{02} r_{2}^{2}\right. \\
& \cdot \frac{\omega_{2}}{3}\left\{3\left(x_{1003}^{2}+y_{1003}^{2}\right)\right\}^{1 / 2} \\
& \left.\cdot r_{2}\left(r_{1} r_{2}\right)^{1 / 2} \cdot \sin \left(\emptyset_{1}+3 \emptyset_{2}\right)+O\left\{\left(r_{1}+r_{2}\right)^{5 / 2}\right\}\right]
\end{aligned}
$$

Assume

$$
\begin{gathered}
a=\left|C_{20}+3 C_{11}+9 C_{02}\right| \\
\text { and } d=\left|3 \omega_{2}\left(x_{1003}^{2}+y_{1003}^{2}\right)^{1 / 2}\right|
\end{gathered}
$$

Now, for the Hamiltonian of the form equation (63), the stability is decided based on the following theorem:

(1) If for a Hamiltonian of perturbed motion, the inequality

$$
\left(x_{1003}^{2}+y_{1003}^{2}\right)^{1 / 2} \neq 0, \quad \text { and } \mathrm{d}>a
$$

is simultaneously satisfied, then the equilibrium point is unstable.

If the inequality signs in (59) change its position and the Hamiltonian contains no terms of the order higher than the fourth, then the equilibrium point is stable.

(2) If the conditions $\left(x_{1003}^{2}+y_{1003}^{2}\right)^{1 / 2}=0, C_{20}+3 C_{11}+$ $9 C_{02}=0$ are simultaneously satisfied, then the equilibrium point is stable. But if $\left(x_{1003}^{2}+y_{1003}^{2}\right)^{1 / 2}=$ $0, C_{20}+3 C_{11}+9 C_{02} \neq 0$, then the stability will be decided by higher order terms than the fourth involving further resonances. It needs separate investigation.

The values of $C_{20}, C_{11}$, and $C_{02}$ for $\omega_{1}=3 \omega_{2}$ are given as

$$
\begin{aligned}
C_{20} & =\frac{27}{2} \omega_{2}^{2} a_{1}^{4} b_{1}^{4} H_{0400}+\frac{1}{6 \omega_{2}^{2}}\left\{a _ { 1 } ^ { 4 } \left(H_{4000}+c_{1} H_{3100}\right.\right. \\
& \left.\left.+c_{1}^{2} H_{2200}+c_{1}^{3} H_{1300}+c_{1}^{4} H_{0400}\right)\right\}+\frac{1}{2}\left\{a _ { 1 } ^ { 4 } b _ { 1 } ^ { 2 } \left(H_{2200}\right.\right. \\
& \left.\left.+3 c_{1} H_{1300}+6 c_{1}^{2} H_{0400}\right)\right\}-\frac{27}{8} \omega_{2}^{2}\left(x_{0030}^{2}+y_{0030}^{2}\right) \\
& -\frac{3}{2}\left(x_{1020}^{2}+y_{1020}^{2}\right)-\frac{9}{10}\left(x_{0120}^{2}+y_{0120}^{2}\right) \\
& +\frac{1}{2}\left(x_{1011}^{2}+y_{1011}^{2}\right)+\frac{9 \omega_{2}^{2}}{10}\left(x_{0021}^{2}+y_{0201}^{2}\right),
\end{aligned}
$$

$$
\begin{aligned}
C_{11} & =18 \omega_{2}^{2} a_{1}^{2} b_{1}^{2} H_{0400}+\frac{a_{1}^{2} a_{2}^{2}}{3 \omega_{2}}\left\{6 H_{4000}+3\left(c_{1}+c_{2}\right)\right. \\
\cdot & H_{3100}+\left(c_{1}^{2}+4 c_{1} c_{2}+c_{2}^{2}\right) H_{2200}+3 c_{1} c_{2}\left(c_{1}+c_{2}\right) \\
\cdot & \left.H_{1300}+6 c_{1}^{2} c_{2}^{2} H_{0400}\right\} 3 a_{2}^{2} a_{1}^{2} b_{1}^{2}\left(H_{2200}+3 c_{2} H_{1300}\right. \\
& \left.+6 c_{2}^{2} H_{0400}\right)+\frac{a_{2}^{2} a_{1}^{2} b_{2}^{2}}{3}\left\{H_{2200}+3 c_{1} H_{1300}\right. \\
& \left.+6 c_{1}^{2} H_{0400}\right\}-\frac{2}{3}\left(x_{1002}^{2}+y_{1002}^{2}\right)+\frac{3 \omega_{2}^{2}}{10}\left(x_{0012}^{2}\right. \\
& \left.+y_{0012}^{2}\right)-\frac{9 \omega_{2}}{14}\left(x_{0021}^{2}+y_{0021}^{2}\right)-\frac{18}{5}\left(x_{0120}^{2}\right. \\
& \left.+y_{0120}^{2}\right)+2\left(x_{0111} x_{1020}+y_{0111} y_{1020}\right) \\
& -\frac{4}{\omega_{2}}\left(x_{0111} x_{1020}+y_{0111} y_{1020}\right), \\
C_{02} & =\frac{3}{2} \omega_{2}^{2} a_{2}^{2} b_{2}^{2} H_{0400}+\frac{3 a_{2}^{2}}{3 \omega_{2}^{2}}\left(H_{4000}+c_{2} H_{3100}\right. \\
& \left.+c_{2}^{2} H_{2200}+c_{2}^{3} H_{1300}+c_{2}^{4} H_{0400}\right)+\frac{a_{2}^{4} b_{2}^{2}}{2}\left(H_{2200}\right. \\
& \left.+3 c_{2} H_{1300}+6 c_{2}^{2} H_{0400}\right)+\frac{3 \omega_{2}^{2}}{8}\left(x_{0003}^{2}+y_{1002}^{2}\right) \\
& +\frac{6}{\omega_{2}}\left(x_{0201}^{2}+y_{0201}^{2}\right)-\frac{\left(x_{1002}^{2}+y_{1002}^{2}\right)}{6} \\
& \left(x_{0111}^{2}+y_{0111}^{2}\right) \\
2 & \frac{3 \omega_{2}^{2}}{40}\left(x_{0012}^{2}+y_{0012}^{2}\right) .
\end{aligned}
$$

\section{Stability in Nonresonance Case}

Equation (30) gives the coefficient of $S_{3}$ in terms of coefficients of $\mathrm{H}_{3}$ reducing $\overline{\mathrm{H}_{3}}=0$. Also $S_{4}$ in (42) and (44) is chosen, so that $\overline{H_{4}}$ retains only terms in normal form (34). Now $S_{3}$ can be expanded as

$$
\begin{aligned}
S_{3}= & g_{0003} P_{2}^{3}+g_{0030} P_{1}^{3}+g_{0300} Q_{2}^{3}+g_{3000} Q_{1}^{3} \\
& +q_{2100} Q_{1}^{2} Q_{2}+g_{2010} Q_{1}^{2} P_{1}+g_{2001} Q_{2}^{2} P_{2} \\
& +g_{1200} Q_{2}^{2} Q_{1}+g_{0210} Q_{2}^{2} P_{1}+g_{0201} Q_{2}^{2} P_{2} \\
& +g_{1020} P_{1}^{2} Q_{1}+g_{0120} P_{1}^{2} Q_{2}+g_{0021} P_{1}^{2} P_{2} \\
& +g_{1002} P_{2}^{2} Q_{1}+g_{0102} P_{2}^{2} Q_{2}+g_{0021} P_{2}^{2} P_{1} \\
& +g_{1110} Q_{1} Q_{2} P_{1}+g_{1101} Q_{1} Q_{2} P_{2}+g_{1011} Q_{1} P_{1} P_{2} \\
& +g_{0111} Q_{2} P_{1} P_{2} .
\end{aligned}
$$


Substituting the required values in (42), the Hamiltonian in the nonresonant case reduces to the form (57), where the coefficients $C_{20}, C_{11}, C_{02}$ are given as

$$
\begin{aligned}
& C_{20}=h_{2020}^{*}-\frac{3}{\iota \omega_{1}} h_{3000}^{\prime} h_{0030}^{\prime}-\frac{3}{\iota \omega_{1}} h_{2010}^{\prime} h_{1020}^{\prime} \\
& +\frac{1}{\iota\left(2 \omega_{1}-\omega_{2}\right)} h_{0120}^{\prime} h_{2001}^{\prime}-\frac{1}{\iota \omega_{2}} h_{1110}^{\prime} h_{1011}^{\prime} \\
& -\frac{1}{\iota\left(2 \omega_{1}+\omega_{2}\right)} h_{2100}^{\prime} h_{0021}^{\prime} \text {, } \\
& C_{11}=h_{1111}^{*}+\frac{4}{\iota\left(\omega_{1}-2 \omega_{2}\right)} h_{0210}^{\prime} h_{1002}^{\prime} \\
& -\frac{4}{\iota\left(\omega_{1}+2 \omega_{2}\right)} h_{1200}^{\prime} h_{0012}^{\prime} \\
& -\frac{4}{\iota\left(\omega_{1}-2 \omega_{2}\right)} h_{2100}^{\prime} h_{0021}^{\prime} \\
& -\frac{4}{\iota\left(2 \omega_{1}-\omega_{2}\right)} h_{2001}^{\prime} h_{0120}^{\prime}-\frac{2}{\iota \omega_{1}} h_{2010}^{\prime} h_{0111}^{\prime} \\
& -\frac{2}{\iota \omega_{1}} h_{1101}^{\prime} h_{1020}^{\prime}-\frac{2}{\iota \omega_{2}} h_{0201}^{\prime} h_{1011}^{\prime} \\
& -\frac{2}{\iota \omega_{2}} h_{1110}^{\prime} h_{0102}^{\prime} \\
& C_{02}=h_{0202}^{*}-\frac{3}{\iota \omega_{2}} h_{0300}^{\prime} h_{0003}^{\prime}-\frac{3}{\iota \omega_{2}} h_{0102}^{\prime} h_{0201}^{\prime} \\
& -\frac{1}{\iota \omega_{1}-2 \omega_{2}} h_{1002}^{\prime} h_{0210}^{\prime}-\frac{1}{\iota \omega_{1}} h_{1101}^{\prime} h_{0111}^{\prime} \\
& -\frac{1}{\iota \omega_{1}} h_{1200}^{\prime} h_{0012}^{\prime} \text {. }
\end{aligned}
$$

Here,

$$
\begin{aligned}
& h_{2020}^{*}=-\frac{1}{2} h_{2020}^{\prime}-\frac{3}{2 \omega_{1}^{2}} h_{4000}^{\prime}-\frac{3}{2} \omega_{1}^{2} h_{0040}^{\prime}, \\
& h_{1111}^{*}=\omega_{1} \omega_{2} h_{0022}^{\prime}+\frac{1}{\omega_{1} \omega_{2}} h_{2200}^{\prime}+\frac{\omega_{1}}{\omega_{2}} h_{0220}^{\prime}+\frac{\omega_{1}}{\omega_{2}} h_{2002}^{\prime}, \\
& h_{0202}^{*}=-\frac{3}{2 \omega_{2}^{2}} h_{0004}^{\prime}-\frac{3}{2 \omega_{2}^{2}} h_{0400}^{\prime}-\frac{1}{2} h_{0202}^{\prime} .
\end{aligned}
$$

The values of $h_{\alpha_{1} \alpha_{2} \gamma_{1} \gamma_{2}}^{\prime}$ are defined in the Appendix. Now if we define

$$
D=C_{20} \omega_{2}^{2}+C_{11} \omega_{1} \omega_{2}+C_{02} \omega_{1}^{2}
$$

the stability in this case is analyzed by applying the KAM for the normalized Hamiltonian (32). The first two conditions are satisfied except for the resonance cases, which is dealt with in a separate section.

\section{Numerical Exploration}

To numerically investigate the results obtained in the study in the previous three sections, the values $\left(x_{1002}^{2}+y_{1002}^{2}\right)$ for the system with different values of $\mu$ is tabulated in Table 1 . But it is observed that the value of $y_{1002}^{2}+x_{1002}^{2} \neq 0$ for the various values of perturbing factors considered. Hence, the motion is unstable for small values of eccentricity $e$ in third order resonance. Similarly, for studying the fourth order resonance case, the values of $a$ and $d$ are presented in Table 2. It is found that the inequalities $d<a$ and $d>a$ are satisfied giving rise to unstable and stable motion depending on the values of $\mu$ and for small values of $e$. For verifying the third condition obtaining the values of $D$. It is clear from Table 3 that the value of $D \neq 0$ for all values of $q_{1}, q_{2}, A_{1}, A_{2}, A_{2}$ and $A_{3}$ and $D<0$ consistently; that is, any possibility that within the assumed values $D$ will vanish at any point does not arise. Hence, the equilibrium points are stable.

Motion of an infinitesimal of assumed oblateness around two binary systems, Luyten 726 and Sirius, has also been explored numerically, by evaluating the deciding factors discussed in the previous sections. The data related to the two binary systems used in the calculation are presented in Table 4.

For both the binary systems the oblateness of both the primaries are assumed to be 0.001 , whereas radiation pressure $q_{1}=0.99$ and $q_{2}=0.98$ and values of all the deciding factors are given in Table 5 .

\section{Discussion and Conclusion}

The nonlinear stability of the elliptical restricted three-body problem with radiating and oblate primaries and infinitesimal satellite has been analyzed. The character of motion is analyzed in the presence as well as in the absence of resonance. If $x_{1002}^{2}+y_{1002}^{2}=0$ and $C_{20}+C_{11}+4 C_{02} \neq 0$ then, by virtue of Markeev's theorem (Markeev, 1967), the equilibrium is stable for third order resonance corresponding to $\omega_{1}=2 \omega_{2}$. But, it is observed that for no value of $q_{1}, q_{2}, A_{1}, A_{2}$, and $A_{3}$, the value of $x_{1002}^{2}+y_{1002}^{2}=0$, which is clear from Table 2 . Hence, the motion is unstable for small values of eccentricity " $e$ " in third order resonance.

In the resonance cases of fourth order corresponding to $\omega_{1}=3 \omega_{2}$, for different values of $q_{1}, q_{2}, A_{1}, A_{2}$, and $A_{3}$, the values of a and d defined by (58) have been calculated. It is found that the inequality $d<a$ is satisfied giving rise to stable motion depending on the values of $q_{1}, q_{2}, A_{1}, A_{2}$, and $A_{3}$ and for small values if $\mathrm{e}$ is as given in Table 3 .

On the other hand, when resonance is not present the values of term $D$, defined by (64), $D \neq 0$, which is clear from Table 1. Thus, it can be concluded that the motion is stable in nonresonance case by the use of KAM theorem.

It is observed that for both the binary systems the movement of infinitesimal in 1:2 resonance shows instable characteristic. However, system Luyten-726 shows stable behavior for 1:3 resonance whereas the values of $a$ and $d$ in the case of binary system Sirius suggest that the system will be instable even in the fourth order resonance. In case of nonresonant movement, it was found that on changing the 
TABLE 1: Values of $x_{1002}^{2}+y_{1002}^{2}$ for third order resonance case.

\begin{tabular}{lcccccccccc}
\hline$\mu$ & $\mathrm{e}$ & $A_{1}$ & $A_{2}$ & $A_{3}$ & $\beta_{1}$ & $\beta_{2}$ & $\alpha$ & $x_{1002}$ & $y_{1002}$ & $x_{1002}^{2}+y_{1002}^{2}$ \\
\hline 0.01 & 0.02 & 0.001 & 0.001 & 0.001 & 0.0002 & 0.0001 & 0.0001 & -0.41720 & -6.19915 & 38.6035 \\
0.02 & 0.02 & 0.001 & 0.001 & 0.001 & 0.0002 & 0.0002 & 0.0001 & -0.69421 & -4.84432 & 23.9494 \\
0.03 & 0.02 & 0.0001 & 0.0001 & 0.0001 & 0.002 & 0.001 & 0.001 & -1.1825 & -3.96232 & 17.0983 \\
0.0001 & 0.016 & 0.001 & 0.001 & 0.001 & 0.0002 & 0.0001 & 0.001 & -200.227 & -975.62 & 991925 \\
0.000004 & 0.04 & 0.001 & 0.001 & 0.001 & 0.0002 & 0.00001 & 0.0001 & -2665.54 & -10322.6 & $1.13661 \times 10^{8}$ \\
\hline
\end{tabular}

TABLE 2: Values of $a$ and $d$ for fourth order resonance case.

\begin{tabular}{lcccccccccc}
\hline$\mu$ & $\mathrm{e}$ & $A_{1}$ & $A_{2}$ & $A_{3}$ & $\beta_{1}$ & $\beta_{2}$ & $\alpha$ & $a$ & $d$ & Nature \\
\hline 0.01 & 0.02 & 0.001 & 0.001 & 0.001 & 0.0002 & 0.0001 & 0.0001 & $1.15522 \times 10^{4}$ & $2.86723 \times 10^{3}$ & Stable \\
0.02 & 0.02 & 0.001 & 0.001 & 0.001 & 0.0002 & 0.0002 & 0.0001 & $2.15443 \times 10^{6}$ & $2.22344 \times 10^{5}$ & Stable \\
0.03 & 0.02 & 0.0001 & 0.0001 & 0.0001 & 0.002 & 0.001 & 0.001 & $2.33605 \times 10^{6}$ & $1.01561 \times 10^{6}$ & Stable \\
0.0001 & 0.016 & 0.001 & 0.001 & 0.001 & 0.0002 & 0.0001 & 0.001 & $2.53384 \times 10^{3}$ & $1.26845 \times 10^{3}$ & Stable \\
\hline
\end{tabular}

TABLE 3: Values of D for nonresonance case.

\begin{tabular}{lcccccccccc}
\hline$\mu$ & $\mathrm{e}$ & $A_{1}$ & $A_{2}$ & $A_{3}$ & $\beta_{1}$ & $\beta_{2}$ & $\alpha$ & $\omega_{1}$ & $\omega_{2}$ \\
\hline 0.01 & 0.02 & 0.001 & 0.001 & 0.001 & 0.0002 & 0.0001 & 0.0001 & 0.697033 & 0.208148 & -109.123 \\
0.02 & 0.02 & 0.001 & 0.001 & 0.001 & 0.0002 & 0.0002 & 0.0001 & 0.68135 & 0.205552 & -49.9179 \\
0.03 & 0.02 & 0.0001 & 0.0001 & 0.0001 & 0.002 & 0.001 & 0.001 & 0.568721 & 0.39769 & -169.405 \\
0.0001 & 0.016 & 0.001 & 0.001 & 0.001 & 0.0002 & 0.0001 & 0.001 & 0.664444 & 0.020441 & -30022.702 \\
0.000004 & 0.04 & 0.001 & 0.001 & 0.001 & 0.0002 & 0.00001 & 0.0001 & 0.677762 & 0.003967 & $-2.27342 \times 10^{4}$ \\
\hline
\end{tabular}

TABLE 4: Data related to binary systems.

\begin{tabular}{lcccc}
\hline Binary System & $M_{1}\left(M_{\odot}\right)$ & $M_{2}\left(M_{\odot}\right)$ & $a(A U)$ & \\
\hline Luyten-726 & 0.101 & 0.99 & 1.95 & \\
\hline Sirius & 2.15 & 1.05 & 7.5 & 0.62 \\
\hline
\end{tabular}

TABLE 5

\begin{tabular}{lccccc}
\hline Binary System & $A_{3}$ & $x_{1002}^{2}+y_{1002}^{2}$ & $a$ & $d$ & 1 \\
\hline \multirow{3}{*}{ Luyten-726 } & 0 & $1.33 \times 10^{8}$ & $4.30 \times 10^{10}$ & $1.80 \times 10^{10}$ & $1.05 \times 10^{8}$ \\
& 0.001 & $1.33 \times 10^{7}$ & $4.01 \times 10^{10}$ & $2.00 \times 10^{10}$ & $-8.48 \times 10^{10}$ \\
& 0.01 & $6.11 \times 10^{7}$ & $3.46 \times 10^{10}$ & $2.12 \times 10^{10}$ & $9.28 \times 10^{8}$ \\
Sirius & 0 & $5.03 \times 10^{8}$ & $4.67 \times 10^{8}$ & $1.81 \times 10^{10}$ & $-1.83 \times 10^{10}$ \\
& 0.001 & $6.45 \times 10^{10}$ & $3.34 \times 10^{8}$ & $2.00 \times 10^{10}$ & $1.04 \times 10^{9}$ \\
& 0.01 & $4.88 \times 10^{8}$ & $5.20 \times 10^{8}$ & $2.12 \times 10^{10}$ & $-1.94 \times 10^{10}$ \\
\hline
\end{tabular}

value of $A_{3}$, there is a sign change for both the systems, so there may exist certain values of $A_{3}$ for which $D$ vanishes. Other than those values, the system shows stable behavior in nonresonant case.

\section{Appendix}

The values of $h_{\alpha_{1} \alpha_{2} \gamma_{1} \gamma_{2}}$ in terms of $\left(q_{i}, p_{i}\right)$ are given as

$$
\begin{aligned}
& h_{0030}=a_{1}^{3} b_{1}^{3} H_{0300}, \\
& h_{3000}=a_{1}^{3}\left(H_{3000}+c_{1} H_{2100}+c_{1}^{2} H_{1200}+c_{1}^{3} H_{0300}\right), \\
& h_{1020}=a_{1}^{3} b_{1}^{2}\left(H_{1200}+3 c_{1} H_{0300}\right),
\end{aligned}
$$

$$
\begin{aligned}
& h_{2010}=a_{1}^{3} b_{1}\left(H_{2100}+2 c_{1} H_{1200}+3 c_{1}^{2} H_{0300}\right), \\
& h_{2001}=-a_{1}^{2} a_{2} b_{2}\left(H_{2100}+2 c_{1} H_{1200}+3 c_{1}^{2} H_{0300}\right), \\
& h_{1011}=-2 a_{1}^{2} a_{2} b_{1} b_{2}\left(H_{1200}+3 c_{1} H_{0300}\right), \\
& h_{1110}=2 a_{1}^{2} a_{2} b_{1}\left\{H_{2100}+\left(c_{1}+c_{2}\right) H_{1200}\right. \\
& \left.\quad+3 c_{1} c_{2} H_{0300}\right\}, \\
& h_{0021}=-3 a_{1}^{2} b_{1}^{2} a_{2} b_{2} H_{0300}, \\
& h_{2100}=a_{1}^{2} a_{2}\left\{3 H_{3000}+\left(2 c_{1}+c_{2}\right) H_{2100}\right. \\
& \left.+c_{1}\left(c_{1}+c_{2}\right) H_{1200}+3 c_{1}^{2} c_{2} H_{0300}\right\},
\end{aligned}
$$




$$
\begin{aligned}
& h_{1002}=a_{1} a_{2}^{2} b_{2}^{2}\left(H_{1200}+3 c_{1} H_{0300}\right) \text {, } \\
& h_{1102}=a_{1} a_{2}^{3} b_{2}^{2}\left\{2 H_{2200}+3\left(c_{1}+c_{2}\right) H_{1300}\right. \\
& h_{0210}=a_{1} a_{2}^{2} b_{1}\left\{H_{2100}+2 c_{2} H_{1200}+3 c_{2}^{2} H_{0300}\right\} \text {, } \\
& \left.+12 c_{1} c_{2} H_{0400}\right\} \text {, } \\
& h_{0012}=3 a_{1} a_{2}^{2} b_{1} b_{2}^{2} H_{0300} \text {, } \\
& h_{1200}=a_{1} a_{2}^{2}\left\{3 H_{3000}+\left(c_{1}+2 c_{2}\right) H_{2100}\right. \\
& \left.+c_{2}\left(2 c_{1}+2 c_{2}\right) H_{1200}+3 c_{1} c_{2}^{2} H_{0300}\right\}, \\
& h_{0120}=a_{2} a_{1}^{2} b_{1}^{2}\left(H_{1200}+3 c_{2} H_{0300}\right) \\
& h_{0111}=-2 a_{2}^{2} a_{1} b_{1} b_{2}\left(H_{1200}+3 c_{2} H_{0300}\right) \text {, } \\
& h_{1101}=-2 a_{2}^{2} a_{1} b_{2}\left\{H_{2100}+\left(c_{1}+c_{2}\right) H_{1200}\right. \\
& \left.+3 c_{1} c_{2} H_{0300}\right\} \text {, } \\
& h_{0201}=-a_{2}^{3} b_{2}\left(H_{2100}+2 c_{2} H_{1200}+3 c_{2}^{2} H_{0300}\right) \text {, } \\
& h_{0102}=a_{2}^{3} b_{2}^{2}\left(H_{1200}+3 c_{2} H_{0300}\right) \\
& h_{0003}=-a_{2}^{3} b_{2}^{3} H_{0300} \text {, } \\
& h_{0300}=a_{2}^{3}\left(H_{3000}+c_{2} H_{2100}+c_{2}^{2} H_{1200}+c_{2}^{3} H_{0300}\right) \text {, } \\
& h_{0040}=a_{1}^{4} b_{1}^{4} H_{0400} \text {, } \\
& h_{4000}=a_{1}^{4}\left(H_{4000}+c_{1} H_{3100}+c_{1}^{2} H_{2200}+c_{1}^{3} H_{1300}\right. \\
& \left.+c_{1}^{4} H_{0400}\right) \text {, } \\
& h_{2020}=a_{1}^{4} b_{1}^{2}\left(H_{2200}+3 c_{1} H_{1300}+6 c_{1}^{2} H_{0400}\right) \text {, } \\
& h_{0022}=6 a_{1}^{2} b_{1}^{2} a_{2}^{2} b_{2}^{2} H_{0400} \\
& h_{2200}=a_{1}^{2} a_{2}^{2}\left\{6 H_{4000}+3\left(c_{1}+c_{2}\right) H_{3100}\right. \\
& +\left(c_{1}^{2}+4 c_{1} c_{2}+c_{2}^{2}\right) H_{2200}+3 c_{1} c_{2}\left(c_{1}+c_{2}\right) H_{1300} \\
& \left.+6 c_{1}^{2} c_{2}^{2} H_{0400}\right\} \text {, } \\
& h_{0220}=a_{1}^{2} a_{2}^{2} b_{1}^{2}\left(H_{2200}+3 c_{2} H_{1300}+6 c_{2}^{2} H_{0400}\right) \text {, } \\
& h_{2002}=a_{1}^{2} a_{2}^{2} b_{2}^{2}\left(H_{2200}+3 c_{1} H_{1300}+6 c_{1}^{2} H_{0400}\right) \text {, } \\
& h_{0004}=a_{2}^{4} b_{2}^{4} H_{0400} \text {, } \\
& h_{0400}=a_{2}^{4}\left(H_{4000}+c_{2} H_{3100}+c_{2}^{2} H_{2200}+c_{2}^{3} H_{1300}\right. \\
& \left.+c_{2}^{4} H_{0400}\right) \text {, } \\
& h_{0202}=a_{2}^{4} b_{2}^{2}\left(H_{2200}+3 c_{2} H_{3100}+6 c_{2}^{2} H_{0400}\right) \text {, } \\
& h_{0013}=-4 a_{1} a_{2}^{3} b_{1} b_{2}^{3} H_{0400}, \\
& h_{1300}=a_{1} a_{2}^{3}\left\{4 H_{4000}+\left(c_{1}+3 c_{2}\right) H_{3100}+4 c_{1} c_{2}^{3} H_{0400}\right. \\
& \left.+2 c_{2}\left(c_{1}+c_{2}\right) H_{2200}+c_{2}^{2}\left(3 c_{1}+c_{2}\right) H_{1300}\right\}, \\
& h_{0211}=-2 a_{1} a_{2}^{3} b_{1} b_{2}\left(H_{2200}+3 c_{2} H_{3100}+6 c_{2}^{2} H_{0400}\right) \text {, } \\
& h_{0112}=3 a_{1} a_{2}^{3} b_{1} b_{2}^{2}\left(H_{1300}+4 c_{2} H_{0400}\right) \\
& h_{1003}=-a_{1} a_{2}^{3} b_{2}^{3}\left(H_{1300}+4 c_{1} H_{0400}\right) \text {, } \\
& h_{1102}=-a_{1} a_{2}^{3} b_{2}\left\{3 H_{3100}+2\left(c_{1}+c_{2}\right) H_{2200}\right. \\
& \left.+3 c_{2}\left(2 c_{1}+c_{2}\right) H_{1300}+12 c_{1} c_{2}^{2} H_{0400}\right\} \text {, } \\
& h_{0310}=a_{1} a_{2}^{3} b_{1}\left\{H_{3100}+2 c_{2} H_{2200}+3 c_{2}^{2} H_{1300}\right. \\
& \left.+4 c_{2}^{3} H_{0400}\right\} \text {, } \\
& h_{0030}^{\prime}=\left(h_{0030}-\frac{h_{2010}}{\omega_{1}^{2}}\right)+i\left(\frac{h_{1020}}{\omega_{1}}-\frac{h_{3000}}{\omega_{1}^{3}}\right), \\
& =x_{0030}+i y_{0030}, \\
& h_{1020}^{\prime}=\left(-\frac{1}{3}\left(h_{1020}-\frac{3}{2} \frac{h_{3000}}{\omega_{1}^{2}}\right)\right. \\
& +i\left(\frac{3 \omega_{1}}{2} h_{0030}-\frac{1}{2 \omega_{1}} h_{2011}\right), \\
& =x_{1020}+i y_{1020} \text {, } \\
& h_{1020}^{\prime}=\left(-\frac{1}{3}\left(h_{1020}-\frac{3}{2} \frac{h_{3000}}{\omega_{1}^{2}}\right)\right. \\
& +i\left(\frac{3 \omega_{1}}{2} h_{0030}-\frac{1}{2 \omega_{1}} h_{2011}\right), \\
& =x_{1020}+i y_{1020}, \\
& h_{0120}^{\prime}=\left(-\frac{\omega_{2}}{2}\left(h_{0021}+\frac{h_{1110}}{2 \omega_{1}}+\frac{\omega_{2}}{2 \omega_{1}^{2}} h_{2001}\right)\right. \\
& +i\left(-\frac{1}{2} h_{0120}-\frac{\omega_{2}}{2 \omega_{1}} h_{1011}+\frac{h_{2100}}{\omega_{1} \omega_{2}}\right), \\
& =x_{1011}+i y_{1011} \text {, } \\
& h_{0120}^{\prime}=\left(-\frac{\omega_{2}}{2}\left(h_{0021}+\frac{h_{1110}}{2 \omega_{1}}+\frac{\omega_{2}}{2 \omega_{1}^{2}} h_{2001}\right)\right. \\
& +i\left(-\frac{1}{2} h_{0120}-\frac{\omega_{2}}{2 \omega_{1}} h_{1011}+\frac{h_{2100}}{\omega_{1} \omega_{2}}\right) \\
& =x_{0120}+i y_{0120} \text {, }
\end{aligned}
$$


Advances in Astronomy

13

$$
\begin{aligned}
& h_{1110}^{\prime}=\left(-\omega_{1} h_{0021}-\frac{h_{2001}}{\omega_{1}}\right)+i\left(\omega_{1} \frac{h_{0120}}{\omega_{2}}+\frac{h_{2100}}{\omega_{1} \omega_{2}}\right) \\
& \left.+i\left(\frac{h_{0300}}{\omega_{2}^{3}}-\frac{h_{0102}}{\omega_{2}}\right)\right] \\
& =x_{1011}+i y_{1011} \text {, } \\
& =-\frac{\omega_{2}^{3}}{8}\left[y_{0003}+i x_{0003}\right] \\
& h_{0021}^{\prime}=\left(\frac{h_{0120}}{\omega_{2}}-\frac{h_{1011}}{\omega_{1}}-\frac{h_{2100}}{\omega_{1}^{2} \omega_{2}}\right)+i\left(h_{0021}+\frac{h_{1110}}{\omega_{1} \omega_{2}}\right. \\
& \left.-\frac{h_{2001}}{\omega_{1}^{2}}\right) \\
& h_{2010}^{\prime}=-\frac{\omega_{1}}{2}\left[\left(\frac{3 \omega_{1}}{2} h_{0030}+\frac{h_{0210}}{2 \omega_{1}}\right)\right. \\
& \left.+i\left(-\frac{h_{1020}}{3}-\frac{3 h_{3000}}{2 \omega_{1}^{2}}\right)\right] \\
& =x_{0021}+i y_{0021} \text {, } \\
& h_{1002}^{\prime}=\left(-\frac{\omega_{1}}{2 \omega_{2}}\left(h_{1011}-\frac{h_{1002}}{2}+\frac{h_{1200}}{2 \omega_{2}^{2}}\right)\right. \\
& =\frac{\omega_{1}}{2}\left[y_{1020}+i x_{1020}\right] \\
& +i\left(-\omega_{1} \frac{h_{0012}}{2}-\frac{\omega_{1}}{2 \omega_{2}^{2}} h_{0210}+\frac{h_{1101}}{2 \omega_{2}}\right) \\
& h_{2001}^{\prime}=-\frac{\omega_{1}^{2}}{2 \omega_{2}}\left[\left(-\frac{h_{0120}}{2}-\frac{\omega_{2}}{2 \omega_{1}} h_{1011}+\frac{1}{2 \omega_{1}^{2}} h_{2100}\right)\right. \\
& \left.+i\left(-\frac{\omega_{2}}{2} h_{0021}+\frac{h_{1110}}{2 \omega_{1}}+\frac{\omega_{2}}{2 \omega_{1}^{2}} h_{2001}\right)\right] \\
& h_{0012}^{\prime}=\left(-h_{0012}+\frac{h_{0210}}{\omega_{2}^{2}}-\frac{h_{1101}}{\omega_{1} \omega_{2}}\right)+i\left(\frac{h_{0111}}{\omega_{2}}\right. \\
& =\frac{\omega_{1}^{2}}{2 \omega_{2}}\left[y_{0120}+i x_{0120}\right] \\
& \left.-\frac{h_{1002}}{\omega_{1}}+\frac{h_{1200}}{\omega_{1} \omega_{2}^{2}}\right) \\
& h_{2100}^{\prime}=-\frac{\omega_{1}^{2} \omega_{2}}{8}\left[\left(-h_{0021}+\frac{h_{1110}}{\omega_{1} \omega_{2}}-\frac{h_{2001}}{\omega_{1}^{2}}\right)\right. \\
& =x_{0012}+i y_{0012} \\
& h_{0111}^{\prime}=\left(-\frac{\omega_{2}}{\omega_{1}}\left(h_{1002}+\frac{h_{1200}}{\omega_{1} \omega_{2}}\right)+i\left(-\omega_{2} h_{0012}\right.\right. \\
& \left.+i\left(\frac{h_{0120}}{\omega_{2}}-\frac{h_{1011}}{\omega_{1}}-\frac{h_{2100}}{\omega_{1}^{2} \omega_{2}}\right)\right] \\
& =\frac{\omega_{1}^{2} \omega_{2}}{8}\left[y_{0021}+i x_{0021}\right] \\
& \left.-\frac{h_{0210}}{\omega_{2}}\right) \\
& =x_{0111}+i y_{0111} \text {, } \\
& h_{0210}^{\prime}=-\frac{\omega_{2}^{2}}{2 \omega_{1}}\left[\left(-\frac{\omega_{1}}{2} h_{0012}+\frac{\omega_{1}}{2 \omega_{2}^{2}} h_{0210}+\frac{h_{1101}}{2 \omega_{2}}\right)\right. \\
& h_{0210}^{\prime}=\left(-\frac{\omega_{2}}{4}\left(h_{0120}-\frac{3}{4 \omega_{2}} h_{0300}\right)+i\left(\frac{3}{4} \omega_{2}^{2} h_{0003}\right.\right. \\
& \left.+i\left(-\frac{\omega_{1}}{2 \omega_{2}} h_{0111}-\frac{h_{1002}}{2}+\frac{h_{1200}}{2 \omega_{2}^{2}}\right)\right] \\
& \left.+\frac{h_{0201}}{4}\right) \\
& =x_{0201}+i y_{0201} \\
& h_{0003}^{\prime}=\left(-\frac{h_{0120}}{\omega_{2}}+\frac{h_{0300}}{\omega_{2}^{3}}\right)+i\left(-h_{0003}+\frac{h_{0201}}{\omega_{2}^{2}}\right) \\
& =x_{0003}+i y_{0003} \\
& h_{3000}^{\prime}=-\frac{\omega_{1}^{3}}{8}\left[\left(\frac{h_{1020}}{\omega_{1}}-\frac{h_{3000}}{\omega_{1}^{3}}\right)\right. \\
& =-\frac{\omega_{2}^{2}}{2 \omega_{1}}\left[y_{0021}+i x_{0021}\right] \\
& h_{1200}^{\prime}=-\frac{\omega_{1} \omega_{2}^{2}}{8}\left[\left(\frac{h_{0111}}{\omega_{2}}-\frac{h_{1002}}{\omega_{1}}+\frac{h_{1200}}{\omega_{1} \omega_{2}^{2}}\right)\right. \\
& \left.+i\left(-h_{0012}+\frac{h_{0210}}{\omega_{2}^{2}}-\frac{h_{1101}}{\omega_{1} \omega_{2}}\right)\right] \\
& =-\frac{\omega_{1} \omega_{2}^{2}}{8}\left[y_{0012}+i x_{0012}\right] \\
& \left.+i\left(h_{0030}-\frac{h_{2010}}{\omega_{1}^{2}}\right)\right] \\
& =-\frac{\omega_{1}^{3}}{8}\left[y_{0030}+i x_{0030}\right] \\
& h_{0300}^{\prime}=-\frac{\omega_{2}^{3}}{8}\left[\left(\frac{h_{0201}}{\omega_{2}^{2}}-h_{0003}\right)\right. \\
& h_{1101}^{\prime}=-\frac{\omega_{1}}{2}\left[\left(-\omega_{2} h_{0012}-\frac{h_{0210}}{\omega_{2}}\right)\right. \\
& \left.+i\left(\frac{\omega_{2}}{\omega_{1}} h_{1002}+\frac{h_{1200}}{\omega_{1} \omega_{2}}\right)\right] \\
& =\frac{\omega_{1}}{2}\left[y_{0111}+i x_{0111}\right]
\end{aligned}
$$




$$
\begin{aligned}
& h_{1110}^{\prime}=\frac{\omega_{2}}{2}\left[\left(\frac{\omega_{1}}{\omega_{2}} h_{0120}+\frac{h_{2100}}{\omega_{1} \omega_{2}}\right)\right. \\
& \left.+i\left(-\omega_{1} h_{0021}-\frac{h_{2001}}{\omega_{1}}\right)\right] \\
& =\frac{\omega_{2}}{2}\left[y_{1011}+i x_{1011}\right] \\
& h_{0120}^{\prime}=\frac{2}{\omega_{2}}\left[\left(\frac{3}{4} \omega_{2}^{2} h_{0003}+\frac{h_{0201}}{4}\right)\right. \\
& \left.\quad+i\left(\omega_{2} \frac{h_{0102}}{4}-\omega_{2} h_{0300}\right)\right] \\
& =\frac{2}{\omega_{2}}\left[y_{0201}+i x_{0201}\right]
\end{aligned}
$$

\section{Data Availability}

No data were used to support this study.

\section{Conflicts of Interest}

The authors declare that they have no conflicts of interest.

\section{Acknowledgments}

The financial assistance from Chattisgarh Council of Science and Technology (Endt. no. 2260/CCOST/MRP/2015) is duly acknowledged with gratitude.

\section{References}

[1] A. P. Markeev, "Libration points in celestial mechanics and astro dynamics," Moscow Izdate Nauka, vol. 1, p. 1, 1978.

[2] J. Györgyey, "On the non-linear stability of motions around L5 in the elliptic restricted problem of the three bodies," Celestial Mechanics and Dynamical Astronomy, vol. 36, no. 3, pp. 281-285, 1985.

[3] K. Goździewski, A. J. Maciejewski, and Z. Niedzielska, "About stability of libration points in the restricted photogravitational three body problem," Celestial Mechanics and Dynamical Astronomy, vol. 52, no. 2, pp. 195-201, 1991.

[4] P. V. Subba Rao and R. K. Sharma, "Effect of oblateness on the non-linear stability of $\mathrm{L}_{4}$ in the restricted three body problem," Celestial Mechanics \& Dynamical Astronomy, vol. 65, no. 3, pp. 291-312, 1996.

[5] A. P. Thakur and R. B. Singh, "Stability of the triangular libration points of the circular restricted problem in the presence of resonances," Celestial Mechanics and Dynamical Astronomy, vol. 66, no. 2, pp. 191-202, 1996.

[6] C. Beauge, S. Ferraz-Mello, and T. A. Michtchenko, "Extrasolar planes in mean motion resonance, apses alignment and asymmetric stationary solutions," The Astrophysical Journal, vol. 593, no. 2, pp. 11-24, 2003.

[7] S. Ferraz-Mello, "Averaging the elliptic asteroidal problem near a first-order resonance," The Astronomical Journal, vol. 94, no. 1, pp. 208-213, 1987.
[8] J. Henrard and N. Caranicolas, "A perturbative treatment of the 2:1 Jovian resonance," ICARUS, vol. 69, no. 2, pp. 266-279, 1987.

[9] J. Henrard, "A semi-numerical perturbation method for separable Hamiltonian system," Celestial Mechanics and Dynamical Astronomy, vol. 49, no. 1, pp. 43-67, 1990.

[10] V. Kumar and R. Choudhry, "Non Linear stability of the triangular liberation points for photogravitational elliptic restricted problem of three bodies," Celestial Mechanics and Dynamical Astronomy, vol. 48, no. 4, pp. 299-317, 1990.

[11] J. D. Hadjidemetriou, "The elliptic restricted problem at the 3: 1 resonance," Celestial Mechanics and Dynamical Astronomy, vol. 53, no. 2, pp. 151-183, 1992.

[12] J. Hadjidemetriou, "Resonant motion in the restricted three body problem," in Qualitative and Quantitative Behaviour of Planetary Systems, R. Dvorak and J. Henrard, Eds., pp. 201-219, Springer, 1993.

[13] L. Floria, "On an analytical solution in the planar elliptical restricted three body problem," Monografsem. Mat. Garacia de Galdeano, vol. 31, pp. 135-144, 2004.

[14] N. Chandra and R. Kumar, "Effect of oblateness on the non-linear stability of the triangular liberation points of the restricted three-body problem in the presence of resonances," Astrophysics and Space Science, vol. 291, no. 1, pp. 1-19, 2004.

[15] A. Narayan and C. Ramesh, "Stability of triangular equilibrium points in elliptic restricted three body problem under the effects of photo gravitation and oblateness of the primaries," International Journal of Pure and Applied Mathematics, vol. 70, pp. 735-754, 2011.

[16] N. Singh and A. Narayan, "Nonlinear stability of the triangular libration points for radiating and oblate primaries in CR3BP in nonresonance condition," Advances in Astronomy, vol. 2015, Article ID 850252, 14 pages, 2015.

[17] A. Narayan, A. Chakraborty, and A. Dewangan, "Dynamics of oblate test particle under the influence of oblate and radiating primaries in elliptic restricted three body problem," Journal of Informatics and Mathematical Sciences, vol. 10, no. 1-2, pp. 7392, 2018.

[18] A. Narayan, A. Chakraborty, and A. Dewangan, "Existence of resonance stability of triangular equilibrium points for an oblate infinitesimal in elliptical restricted three body problem," $M M C$ B, AMSE Journals, vol. 86, no. 3, pp. 609-626, 2017. 

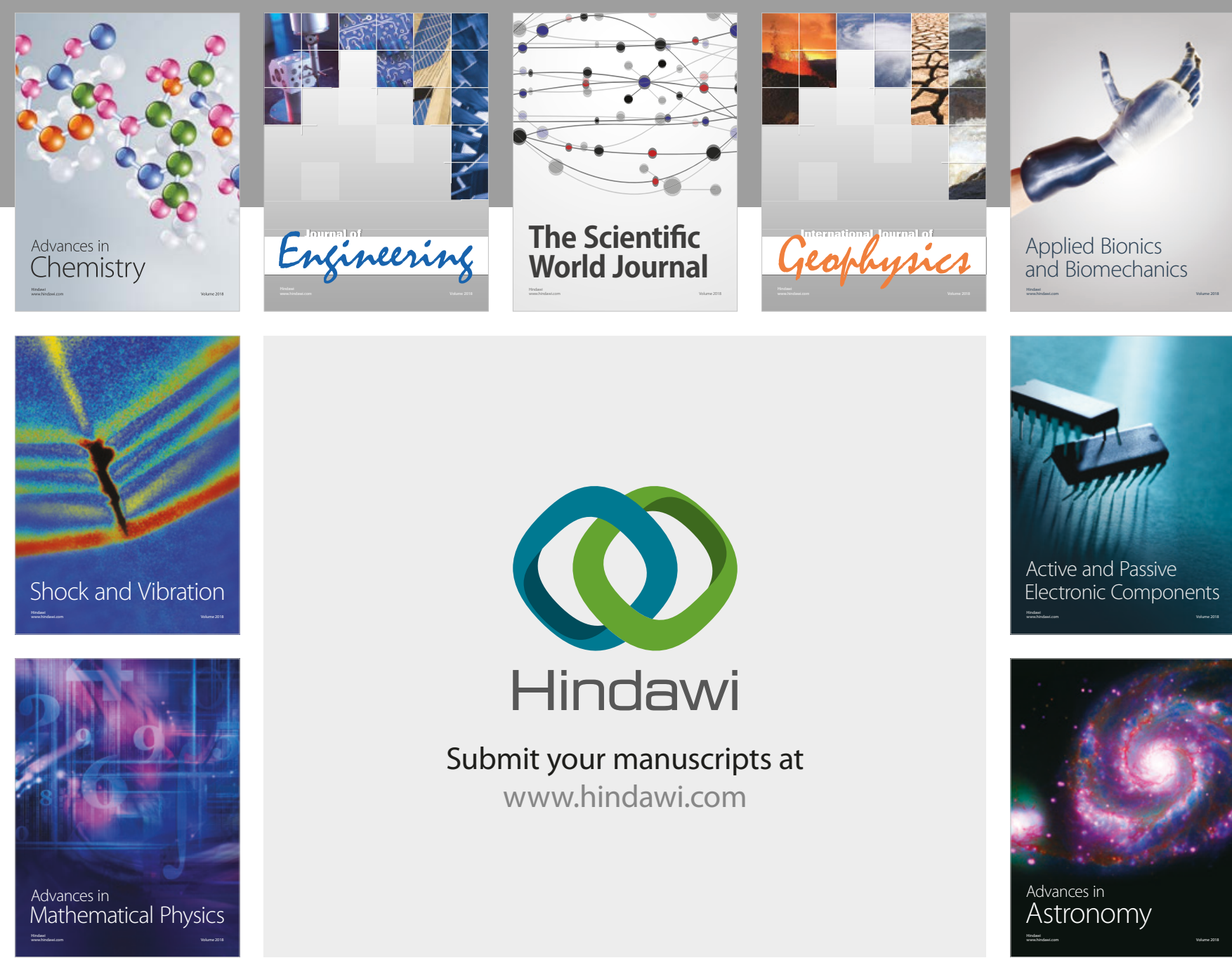

Submit your manuscripts at

www.hindawi.com

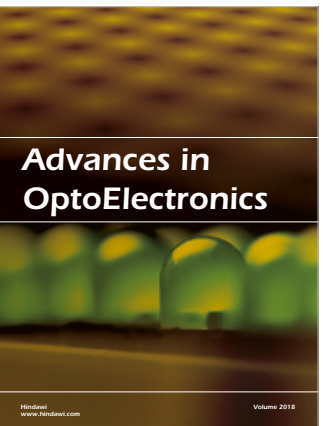

\section{Rotcting Machinery}
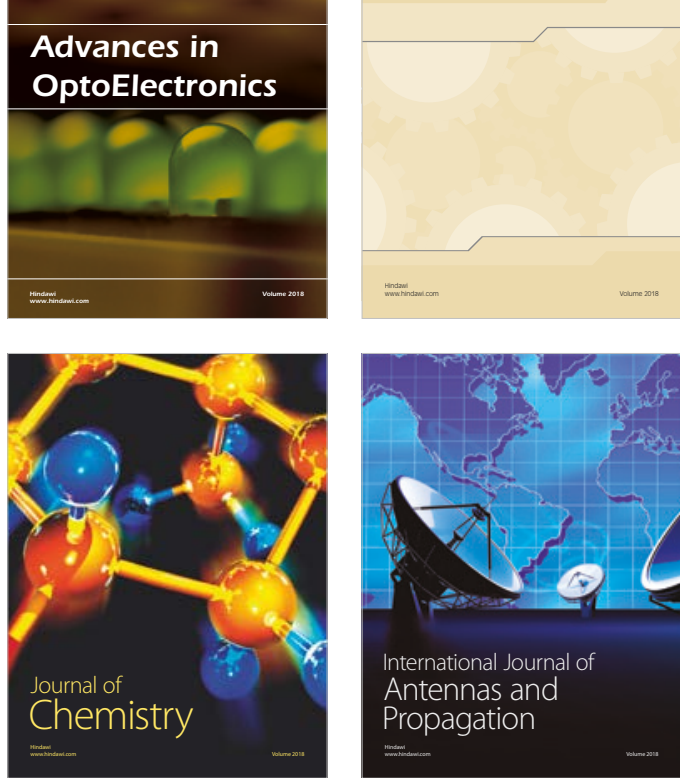

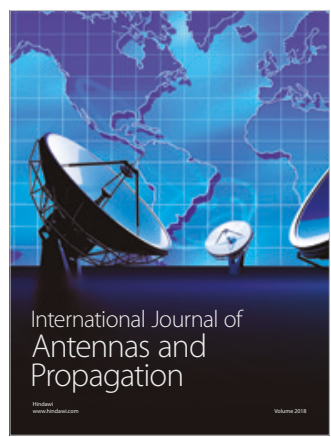

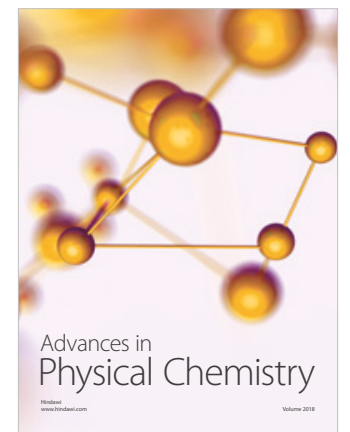

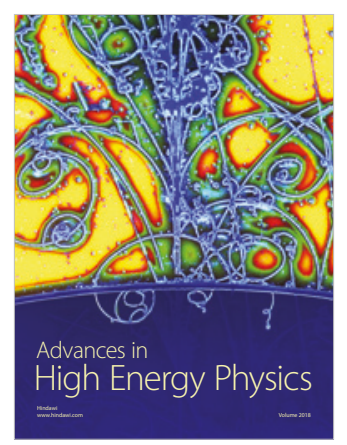

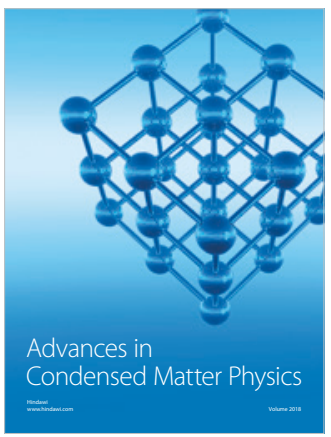

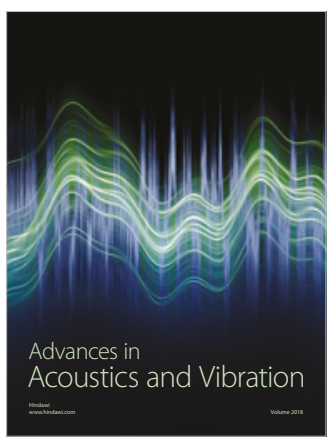

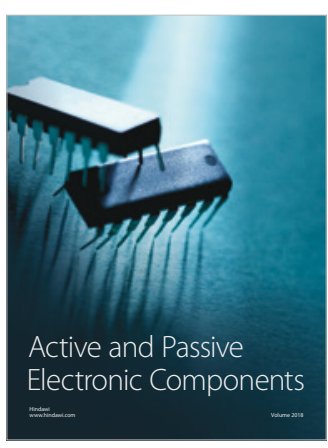
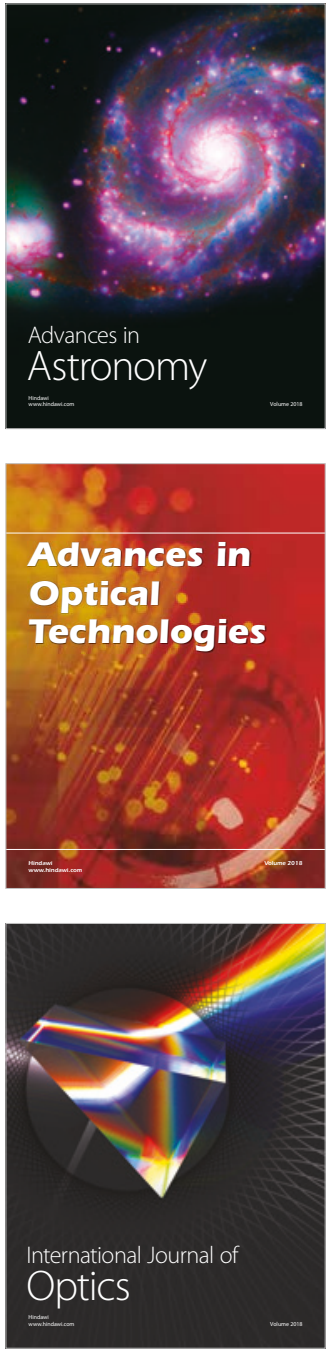